\title{
Colloquium: Quantum annealing and analog quantum computation
}

\author{
Arnab Das* and Bikas K. Chakrabarti ${ }^{\dagger}$ \\ Theoretical Condensed Matter Physics Division and Centre for Applied Mathematics and \\ Computational Science, Saha Institute of Nuclear Physics, 1/AF, Bidhannagar, \\ Kolkata-700064, India
}

(Published 5 September 2008)

\begin{abstract}
The recent success in quantum annealing, i.e., optimization of the cost or energy functions of complex systems utilizing quantum fluctuations is reviewed here. The concept is introduced in successive steps through studying the mapping of such computationally hard problems to classical spin-glass problems, quantum spin-glass problems arising with the introduction of quantum fluctuations, and the annealing behavior of the systems as these fluctuations are reduced slowly to zero. This provides a general framework for realizing analog quantum computation.
\end{abstract}

DOI: 10.1103/RevModPhys.80.1061 PACS number(s): 02.10.Ox, 02.60.Pn, 02.70.Ss, 03.65.Xp

\section{CONTENTS}

I. Introduction

II. Optimization and Annealing

A. Combinatorial optimization problems

B. Statistical mechanics of the optimization problems and thermal annealing

C. Spin glasses and optimization

1. Finding the ground states of classical spin glasses

2. The traveling salesman problem

D. Quantum spin glasses and annealing

III. Quantum Annealing

A. Quantum Monte Carlo annealing

1. A short-range spin glass

2. The traveling salesman problem

3. Random field Ising model: How the choice of kinetic term improves annealing results

B. Quantum annealing using real-time adiabatic evolution

C. Annealing of a kinetically constrained system

D. Experimental realization of quantum annealing

IV. Convergence of Quantum Annealing Algorithms

V. Quantum Quenching

VI. Summary And Discussions

Acknowledgments

Appendix A

1. Suzuki-Trotter formalism

2. Quantum quenching of a long-range TIM

References

\section{INTRODUCTION}

The use of quantum-mechanical tunneling through classically localized states in annealing of glasses has opened up a new paradigm for solving hard optimization

\footnotetext{
*arnab.das@saha.ac.in

†bikask.chakrabarti@saha.ac.in
}

problems through adiabatic reduction of quantum fluctuations. This topic will be introduced and reviewed here.

Consider the example of a ferromagnet consisting of $N$ small interacting magnetic elements: the spins. For a macroscopic sample, $N$ is very large: on the order of the Avogadro number. Assume that each spin can be in either of two simple states: up or down. Also, the pairwise interactions between spins are such that the energy of interaction [potential energy (PE)] between any pair of spins is negative (smaller) if both the spins in the pair are in the same state and positive (larger) if their states differ. Thus, the collective energy of the $N$-spin system (given by the Hamiltonian $\mathcal{H}$ ) is minimum when all spins are aligned in the same direction, all up or all down, giving complete order. We call these two minimumenergy configurations the ground states. The rest of the $2^{N}$ configurations are called excited states. The plot of the interaction energy for the whole system with respect to the configurations is called the potential-energyconfiguration landscape, or simply the potential-energy landscape (PEL). For a ferromagnet, this landscape has a smooth double-valley structure (two mirror-symmetric valleys with the two degenerate ground states, all up and all down, at their respective bottoms). At zero temperature the equilibrium state is the state of minimum potential energy, and the system resides stably at the bottom of either of the two valleys. At finite temperature, thermal fluctuations allow the system to visit higher-energy configurations with some finite probability (given by the Boltzmann factor) and thus the system also spends time in other parts of the PEL. The probability that a system is found in a particular macroscopic state depends not only on the energy of the state (as at zero temperature), but also on its entropy. The thermodynamic equilibrium state corresponds to the minimum of a thermodynamic potential called the free energy $F$, given by the difference between the energy of the state and the product of its entropy and the temperature. At zero temperature, the minimum of the free energy coincides with the minimum for energy and one gets the highest order (magne- 
tization). As the temperature is increased, the contribution of the entropy is magnified and the minimum of the free energy is shifted more and more toward states with lower and lower order or magnetization, until at (and beyond) some transition temperature $T_{c}$ the order disappears completely. For antiferromagnetic systems, the spin-spin pair interactions are such that the energy is lower if the spins in the pair are in opposite states, and higher if their states are same. For antiferromagnets one can still define a sublattice order or magnetization and the PEL still has a double-well structure as in a ferromagnet for short-range interactions. The free energy and the order-disorder transition also show identical behavior as observed for a ferromagnet.

In spin glasses, where different spin-spin interactions are randomly ferromagnetic or antiferromagnetic and frozen in time (quenched disorder), the PEL becomes extremely rugged; various local and global minima trapped between potential-energy barriers appear. The ruggedness and degeneracies in the minima come from the effect of frustration or competing interactions between spins; none of the spin states on a cluster or a plaquette is able to satisfy all interactions in the cluster. The locally optimal state for spins in the cluster is therefore degenerate and frustrated.

Similar situations occur for multivariate optimization problems such as the traveling salesman problem (TSP). Here a salesman has to visit $N$ cities placed randomly on a plane (country). Of the $N ! / N$ distinct tours passing through each city once, only a few correspond to the minimum (ground-state) travel distance or travel cost. The rest correspond to higher costs (excited states). The cost function, when plotted against different tour configurations, gives a similar rugged landscape, equivalent to the PEL of a spin glass (henceforth we use the term PEL to also mean cost-configuration landscapes).

Obviously, an exhaustive search for the global minimum of a rugged PEL requires an exponentially large (or higher) number (in $N$ ) of searches $\left(2^{N}\right.$ and $N$ ! order of searches for an $N$-spin spin glass and an $N$-city TSP, respectively). The computational effort or time for such searches is therefore generally not bounded by any polynomial in the problem size $N$. Alternatively, a gradual energy or cost dissipative dynamics (annealing) with Boltzmann-like thermal fluctuations or some noise factor (in order to move beyond the local minima) in the PEL may help deep enough minima to be reached more easily. This simulated thermal annealing scheme is now considered to be a successful technique. However, the technique often fails if barrier heights between minima diverge (or become very high), as in the case of a spin glass, due to frequent trapping of the system in such local minima (glassy behavior). If the barriers are very narrow, quantum-mechanical fluctuations (fluctuations in a quantum observable due to its noncommutativity with the Hamiltonian of the system) can assist in tunneling through them, thereby leading to successful quantum annealing (see Fig. 1). We introduce these ideas in detail here through successive steps:

(i) The physics of classical spin glasses has already

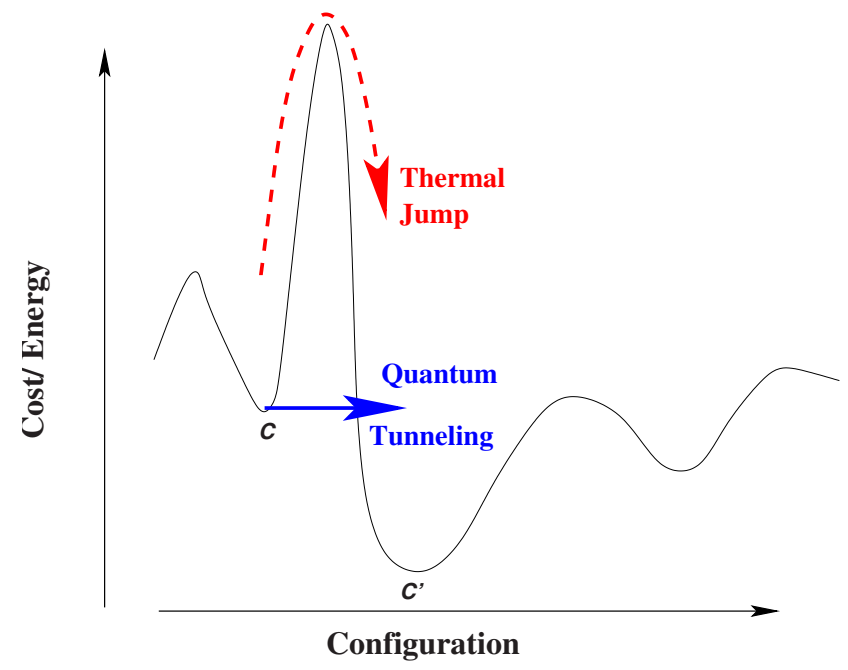

FIG. 1. (Color online) To optimize the cost function of a computationally hard problem (such as the ground-state energy of a spin glass or the minimum travel distance for a traveling salesman problem), one has to get out of a shallower local minimum such as the configuration $C$ (spin configuration or travel route) to reach a deeper minimum $C^{\prime}$. This requires jumps or tunnelinglike fluctuations in the dynamics. Classically, one has to jump over the energy or cost barriers separating them, while quantum mechanically one can tunnel through them. If the barrier is high enough, thermal jumping becomes very difficult. However, if the barrier is narrow enough, quantum tunneling often becomes quite easy.

contributed to our knowledge of the landscape structure of the energy or thermodynamic potential and that of the unusually slow (glassy) dynamics of many-body systems in the presence of frustration and disorder. Mapping of computationally hard problems, such as the traveling salesman problem, etc., to classical spin-glass models also helped in understanding their complexity.

(ii) The ground- (and some low-lying-) state structures of frustrated random systems in the presence of quantum fluctuations have also been studied in the context of quantum spin glasses. It has been shown that, because of the possibility of tunneling through barriers in the potential-energy landscape, quantum fluctuations can help the dynamics to be "more ergodic" than the dynamics induced by classical fluctuations and thus lead to better exploration of the landscape. Ergodicity here means memory loss of the initial state in the course of evolution (weak ergodicity) and convergence to a stationary distribution irrespective of the initial state (strong ergodicity). The nature of these quantum phase transitions in such systems has also been extensively studied. These studies (Sec. II.D) provide knowledge of the phase diagram and the location of the quantum critical point or phase boundary which is crucial for choosing the proper quantum kinetic terms and the annealing path (Secs. III.A and III.B).

(iii) The most natural connection between the paradigm of classical spin glasses and hard optimization problems comes through a widely used and wellestablished optimization technique, namely, the simu- 
lated annealing algorithm as discussed earlier. The possibility of quantum tunneling through classically impenetrable barriers, as indicated by studies of quantum spin glasses, naturally suggests an elegant and often more effective alternative to simulated annealing.

In quantum annealing, one has a classical Hamiltonian (or a multivariate cost function viewed as the same) to be optimized, to which one adds a (noncommuting) quantum kinetic term and reduces it from a high initial value to zero eventually. This reduction, when done completely adiabatically, assures that the ground state of the classical glass is reached at the end, assuming that there is no crossing of energy levels with the ground state in the course of evolution, and provided that the starting state was the ground state of the initial Hamiltonian. To start with, the tunneling field is much higher than the interaction term, so the ground state (a uniform superposition of all classical configurations) is trivially realizable. Simulations demonstrate that quantum annealing can occasionally help reach the ground state of a complex glassy system much faster than done using thermal annealing (discussed later in Sec. III). An experiment comparing classical and quantum annealing for a spin glass also shows that the relaxations in the course of quantum annealing are often much faster than those during the corresponding classical annealing, as discussed in Sec. III.D. What makes quantum annealing fundamentally different from classical annealing is the nonlocal nature (Sec. III) and its higher tunneling ability (Secs. II.D and III.C).

Quantum annealing thus permits a realization of ana$\log$ quantum computation, which is an independent and powerful complement to digital quantum computation, where discrete unitary transformations are implemented through quantum logic gates.

\section{OPTIMIZATION AND ANNEALING}

\section{A. Combinatorial optimization problems}

The occurrence of multivariate optimization problems is ubiquitous in our life, wherever one has to choose the best bargain from a host of available options that depend on many independent factors. In many cases, such a task can be cast as a problem of minimizing a given cost or energy function $\mathcal{H}\left(S_{1}, S_{2}, \ldots, S_{N}\right)$ with respect to $N$ variables $S_{1}, S_{2}, \ldots, S_{N}$ (sometimes subject to some constraints). The task is to find a set of values for these variables (a configuration) for which the function $\mathcal{H}\left(\left\{S_{i}\right\}\right)$ has the minimum value (cf. Fig. 1). In many important optimization problems, the set of feasible configurations from which an optimum is to be chosen is a finite set (for finite $N$ ). In such a case, we say that the problem is combinatorial in nature. If the variables $S_{i}$ are discrete and each takes a finite number of values, then the problem is a combinatorial one. Moreover, certain problems with continuous variables (such as linear programming problems) can also be reduced to combinatorial problems (Papadimitriou and Steiglitz, 1998). Here we focus on this type of optimization problem, and assume that we have to minimize $\mathcal{H}\left(\left\{S_{i}\right\}\right)$ with respect to the discrete set of variables $S_{i}$.

An optimization problem is said to belong to the class $P$ ( $P$ for polynomial), if it can be solved in polynomial time (i.e., the evaluation time varies as some polynomial in $N$ ) using polynomially (in $N$, again) bound resources (computer space, processors, etc.). The existence of such a polynomial bound on the evaluation time is sometimes interpreted as the "easiness" of the problem. However, many important optimization problems seem to fall outside this class, such as the traveling salesman problem (see Sec. II.C.2).

There is another important class of problems which can be solved in polynomial time by nondeterministic machines. This class is the nondeterministic polynomial (NP) class (Garey and Johnson, 1979). $P$ is included completely in the NP class, since a deterministic Turing machine is a special case of nondeterministic Turing machines. Unlike a deterministic machine, which takes a specific step deterministically at each instant (and hence follows a single computational path), a nondeterministic machine has a host of different "allowed" steps at its disposal at every instant. At each instant it explores all allowed steps and if any one of them leads to the goal, the job is considered to be done. Thus it explores in parallel many paths (whose number varies roughly exponentially with time) and checks if any one of them reaches the goal.

Among the NP problems, there are certain problems (known as NP-complete problems) which are such that any NP problem can be "reduced" to them using a polynomial algorithm. The famous 3-SAT problem (see Sec. III.A.3) is a representative of the class. This roughly means that, if one has a routine to solve an NP-complete problem of size $N$, then using that routine one can solve any NP problem at the cost of an extra overhead in time that varies only polynomially with $N$. Problems in this class are considered to be hard, since so far a general nondeterministic machine cannot be simulated by a deterministic Turing machine (or any sequential computer with polynomially bound resources) without an exponential growth of execution time. In fact, it is widely believed (though not proved yet) that it is impossible to do so (i.e., $P \neq \mathrm{NP}$ ) in principle. However, assuming this to be true, one can show that there are indeed problems in the NP class that are neither NP complete nor $P$ (Garey and Johnson, 1979).

\section{B. Statistical mechanics of the optimization problems and thermal annealing}

There are some excellent deterministic algorithms for solving certain optimization problems exactly $(\mathrm{Pa}-$ padimitriou and Steiglitz, 1998; Hartmann and Rieger, 2002). These algorithms are, however, small in number and are strictly problem specific. For NP or harder problems, only approximate results can be found using these algorithms in polynomial time. These approximate algorithms too are also strictly problem specific, in the sense 
that, if one can solve a certain NP-complete problem up to a certain approximation using some polynomial algorithm, that does not ensure that one can solve all other NP problems using the same algorithm up to the said approximation in polynomial time.

Exact algorithms being scarce, one has to look for heuristic algorithms, which are algorithms based on certain intuitive moves, without any guarantee on either the accuracy or the run time for the worst case instance. However, these algorithms are generally easy to formulate and are effective in solving most instances of the intended problems. A general approach toward formulating such approximate heuristics may be based on stochastic (randomized) iterative improvements. The most common starting point in this family is the local minimization algorithm. In this algorithm one starts with a random configuration $C_{0}$ and makes some local changes in the configuration following some prescription (stochastic or deterministic) to generate a new configuration $C_{1}$ and calculates the corresponding change in the cost. If the cost is lowered by the change, then the new configuration $C_{1}$ is adopted. Otherwise the old configuration is retained. Then in the next step a new local change is attempted again, and so on. This reduces the cost steadily until a configuration is reached that minimizes the cost locally. This means that no further lowering of cost is possible by changing this configuration using any of the prescribed local moves. The algorithm essentially stops there. But generally, in most optimization problems (as in spin glasses), there occur many local minima in the cost-configuration landscape and they are mostly far above the global minimum (see Fig. 1). It is likely that the algorithm therefore gets stuck in one of them and ends up with a poor approximation. One can then start afresh with some new initial configuration and end up with another local minimum. After repeating this several times, each time with a new initial configuration, one may choose the best result from them. But a much better idea would be to somehow get out of shallow local minima. One can introduce some fluctuations or noise in the process so that the movement is not always toward lower-energy configurations, but there is also a finite probability to go to higher-energy configurations (the higher the final state energy, the lower the probability to move there), and consequently chances appear to get out of the shallow local minima. Initially, strong fluctuations are adopted (i.e., the probability to go to higher-energy configurations is relatively high) and slowly the fluctuations are reduced until finally they are turned off completely. In the meantime the system gets a fair opportunity to explore the landscape more exhaustively and settle into a reasonably deep cost or energy minimum. Kirkpatrick et al. (1983) suggested an elegant method: A fluctuation is implemented by introducing an "artificial" temperature $T$ into the problem such that the transition probability from a configuration $C_{i}$ to a configuration $C_{f}$ is given by $\min \left\{1, \exp -\left(\Delta_{i f} / T\right)\right\}$, where $\Delta_{i f}$ $=E_{f}-E_{i}$, with $E_{k}$ denoting the cost or energy of the configuration $C_{k}$. A corresponding Monte Carlo dynamics is defined, say, based on detailed balance, and the thermal relaxation of the system is simulated. In the course of simulation, the noise factor $T$ is reduced slowly from a high initial value to zero, following some annealing schedule. At the end of the simulation one is expected to end up with a configuration whose cost is a reasonable approximation of the globally minimum one. If the temperature is decreased slowly enough, say,

$$
T(t) \geq N / \ln t,
$$

where $t$ denotes the cooling time and $N$ is the system size, then the global minimum is attained with certainty in the limit $t \rightarrow \infty$ (Geman and Geman, 1984). Even within a finite time and with a faster cooling rate, one can achieve a reasonably good approximation (a crystal with only a few defects) in practice. This simulated annealing method is now used extensively by engineers for devising real-life optimization algorithms. We refer to this as classical annealing (CA), to distinguish it from quantum annealing (QA) which employs quantum fluctuations. It is important to note that, although in this type of stochastic algorithm the system has many different steps with their corresponding probabilities at its disposal, it finally takes up a single one, chosen, say, by tossing coins, and thus finally follows a single (stochastically selected) path. Hence it is not equivalent to a nondeterministic machine, where all allowed paths are checked in parallel at every time step.

As mentioned already, many combinatorial optimization problems can be cast into the problem of finding the ground state of some classical (spin-glass-like) Hamiltonian $H\left(\left\{S_{i}\right\}\right)$. One can therefore analyze the problem using statistical mechanics to apply physical techniques like simulated annealing. If one naively takes the number of variables $N$ as the size, then the entropy and the energy are often found to scale differently with $N$ and applying standard thermodynamic arguments becomes difficult. One needs to scale temperature and some other quantities properly with $N$ so that one can talk in terms of concepts like free-energy minimization, etc. Moreover, the constraints present in the problems are often difficult to take into account.

\section{Spin glasses and optimization}

\section{Finding the ground states of classical spin glasses}

As mentioned already, the difficulty faced by a physically motivated optimization heuristic (one that follows physical relaxation dynamics, classical or quantum, to search for the solution) in finding the solution of a hard optimization problem is similar to that faced by a glassy system in reaching its ground state. In fact, finding the ground state of a spin glass is an important class of combinatorial optimization problem, which includes an NPcomplete problem (Barahona, 1982), and many other apparently different ones (such as the traveling salesman problem) can be recast in this form. Hence, we discuss here the nature of the spin-glass phase and the difficulty in reaching its ground state. 
The interaction energy of a typically random and frustrated Ising spin glass (Binder and Young, 1986; Dotsenko, 2001; Nishimori, 2001) may be represented by a Hamiltonian of the form

$$
\mathcal{H}=-\sum_{i>j}^{N} J_{i j} S_{i} S_{j},
$$

where $S_{i}$ denote the Ising spins and $J_{i j}$ the interactions between them. The $J_{i j}$ 's here are quenched variables which vary randomly in both sign and magnitude following some distribution $\rho\left(J_{i j}\right)$. The typical distributions are the (zero-mean) Gaussian distribution of positive and negative $J_{i j}$ values,

$$
\rho\left(J_{i j}\right)=A \exp \left(-\frac{J_{i j}^{2}}{2 J^{2}}\right),
$$

and the binary distribution,

$$
\rho\left(J_{i j}\right)=p \delta\left(J_{i j}-J\right)+(1-p) \delta\left(J_{i j}+J\right),
$$

with probability $p$ of having a $+J$ bond, and $1-p$ of having a $-J$ bond. Two well-studied models are the Sherrington-Kirkpatrick (SK) model (Sherrington and Kirkpatrick, 1975) and the Edwards-Anderson (EA) model (Edwards and Anderson, 1975). In the SK model the interactions are infinite ranged and for the sake of extensivity (for the rules of equilibrium thermodynamics to be applicable, the energy should be proportional to the volume, or its equivalent that defines the system size) one has to scale $J \sim 1 / \sqrt{N}$, while in the EA model, the interactions are between nearest neighbors only. For both of them, however, $\rho\left(J_{i j}\right)$ is Gaussian; $A$ $=\left(N / 2 \pi J^{2}\right)^{1 / 2}$ for normalization in the SK model.

Freezing (temperatures below $T_{c}$ ) is characterized by some nonzero value of the thermal average of the magnetization at each site (local ordering). However, since the interactions are random and competing, the spatial average of single-site magnetization (below $T_{c}$ ) is zero. Above $T_{c}$, both the spatial and temporal averages of the single-site magnetization vanish. A relevant order parameter for this freezing to occur is therefore

$$
q=\frac{1}{N} \overline{\sum_{i}^{N}\left\langle S_{i}\right\rangle_{T}^{2}}
$$

[with the overbar denoting the average over disorders (the distribution of $J_{i j}$ ) and $\langle\cdots\rangle_{T}$ denoting the thermal average]. Here $q \neq 0$ for $T<T_{c}$, while $q=0$ for $T \geq T_{c}$. As shown in the following, the existence of a unique order parameter $q$ indicates ergodicity.

In the spin-glass phase $\left(T<T_{c}\right)$, the whole free-energy landscape is divided (cf. Fig. 1) into many valleys (local minima of the free energy) separated by high freeenergy barriers. Thus the system, once trapped in a valley, remains there for a long time. The spins of such a confined system are allowed to explore only a restricted (and correlated) part of the configuration space, and thus "freeze" with a magnetization that characterizes the state (valley) locally.
To date two competing pictures continue to represent the physics of the spin glasses. The mean-field picture of replica symmetry breaking is valid for infinite-ranged spin-glass systems like the SK spin glass. In this picture, below the glass transition temperature $T_{c}$, the barriers separating the valleys in the free-energy landscape actually diverge (in the limit $N \rightarrow \infty$ ), giving rise to a diverging time scale for the confinement of the system in any such valley once the system gets there somehow. This means there is a loss of ergodicity in the thermal dynamics of the system at $T<T_{c}$. Thus one needs a distribution $P(q)$ of order parameters, instead of a single order parameter, to characterize the whole landscape, as emerges naturally from the replica symmetry-breaking ansatz of Parisi (1980). To be a bit more quantitative, imagine that two identical replicas (having exactly the same set of $J_{i j}$ 's) of a spin-glass sample are allowed to relax thermally below $T_{c}$, starting from two different random (paramagnetic) initial states. Then these two replicas (labeled by $\mu$ and $\nu$, say) will settle in two different valleys, each characterized by a local value of the order parameter and the corresponding overlap parameters $q_{\mu \nu}$, which have a sample-specific distribution

$$
\begin{aligned}
& P_{J}(q)=\sum_{\mu, \nu} e^{-\left(F_{\mu}+F_{\nu}\right) / T} \delta\left(q-q_{\mu \nu}\right), \\
& P(q)=\int \prod_{i>j} d J_{i j} \rho\left(J_{i j}\right) P_{J}(q) .
\end{aligned}
$$

Here the subscript $J$ denotes a particular sample with a given realization of quenched random interactions $\left(J_{i j}\right.$ 's) between spins, and finally, by averaging $P_{J}(q)$ over the disorder distribution $\rho(J)$ in Eq. (2) or (3), one gets $P(q)$. Physically, $P(q)$ gives the probability distribution for the two pure states to have an overlap $q$, assuming that the probability of reaching any pure state $\mu$ starting from a random (high-temperature) state is proportional to the thermodynamic weight $\exp \left\{-F_{\mu}\right\}$ of the state $\mu$.

The other picture of the physics of spin glasses is due to the droplet model of short-range spin glasses (Bray and Moore, 1984; Fisher and Huse, 1986), where there is no divergence in the typical free-energy barrier height, and the relevant time scale is taken to be that of crossing the free-energy barrier of formation of a typical droplet of same (all up or all down) spins. Based on a certain scaling ansatz, this picture leads to a logarithmically decaying (with time) self-correlation function for the spins below the freezing temperature $T_{c}$.

The validity of the mean-field picture (of replica symmetry breaking) in the context of real-life spin glasses, where interactions are essentially short range, is far from settled (see, e.g., Marinari et al., 1998; Moore et al., 1998; Krzakala et al., 2001; Gaviro et al., 2006). However, the effective Hamiltonian (cost function) for many other optimization problems may contain long-range interactions and may even show the replica symmetry-breaking behavior shown in the graph partitioning problem (Fu and Anderson, 1986). Of course, no result of QA for such a system (for which replica symmetry breaking is shown 
explicitly) has been reported yet. The successes of QA reported so far are mostly for short-range systems. Thus the scope of quantum annealing in those long-range systems still remains an interesting open question.

\section{The traveling salesman problem}

In the traveling salesman problem, there are $N$ cities placed randomly in a country, with a definite metric to calculate the intercity distances. A salesman has to make a tour to cover every city and finally come back to the starting point. The problem is to find the tour of minimum length. An instance of the problem is given by a set $\left\{d_{i j} ; i, j=1, N\right\}$, where $d_{i j}$ indicates the distance between the $i$ th and $j$ th cities, or equivalently the cost for going from the former to the latter. We mainly focus on the results of the symmetric case, where $d_{i j}=d_{j i}$. The problem can be cast into a form where one minimizes an Ising Hamiltonian under some constraints, as shown below. A tour can be represented by an $N \times N$ matrix $\mathbf{T}$ with elements either 0 or 1 . In a given tour, if the city $j$ is visited immediately after visiting city $i$, then $\mathbf{T}_{i j}=1$ or else $\mathbf{T}_{i j}=0$. Generally, an additional constraint is imposed that each city has to be visited once and only once in a tour. Any valid tour with the above restriction may be represented by a $\mathbf{T}$ matrix whose each row and each column has one and only one element equal to 1 and the rest are all 0's. For a symmetric metric, a tour and its reverse have the same length, and it is more convenient to work with an undirected tour matrix $\mathbf{U}=\frac{1}{2}(\mathbf{T}+\tilde{\mathbf{T}})$, where $\tilde{\mathbf{T}}$, the transpose of $\mathbf{T}$, represents the reverse of the tour given by $\mathbf{T}$. Clearly, $\mathbf{U}$ must be a symmetric matrix having two and only two distinct entries equal to 1 in every row and every column, with no two rows or two columns identical. In terms of the $\mathbf{U}_{i j}$ 's, the length of a tour can be represented by

$$
\mathcal{H}=\frac{1}{2} \sum_{i, j=1}^{N} d_{i j} \mathbf{U}_{i j} .
$$

One can rewrite the above Hamiltonian in terms of Ising spins $S_{i j}$ 's as

$$
\mathcal{H}_{\mathrm{TSP}}=\frac{1}{2} \sum_{i, j=1}^{N} d_{i j} \frac{\left(1+S_{i j}\right)}{2},
$$

where $S_{i j}=2 \mathbf{U}_{i j}-1$ are the Ising spins. The Hamiltonian is similar to that of noninteracting Ising spins on an $N \times N$ lattice, with random fields $d_{i j}$ on the lattice points $\{i, j\}$. The frustration is introduced by the global constraints on the spin configurations in order to conform with the structure of the matrix $\mathbf{U}$ discussed above. The problem is to find the ground state of the Hamiltonian subject to these constraints. There are $N^{2}$ Ising spins, which can assume $2^{N^{2}}$ configurations in the absence of any constraint, but the constraint here reduces the number of valid configurations to the number of distinct tours, which is $(N !) / 2 N$.
Two distinct classes of the TSP are mainly studied, one with a Euclidean $d_{i j}$ in finite dimensions (where the $d_{i j}$ are strongly correlated through triangle inequalities, which means that, for any three cities $A, B$, and $C$, the sum of any two of the sides $A B, B C$, and $C A$ must be greater than the remaining one), and the other with random $d_{i j}$ in infinite dimension.

In the first case, $N$ cities are uniformly distributed within a hypercube in a $d$-dimensional Euclidean space. Finding a good approximation for large $N$ is easier in this case, since the problem is finite ranged. Here a $d$-dimensional neighborhood is defined for each city, and the problem can be solved by dividing the whole hypercube into a number of smaller pieces and then searching for the least path within each smaller part and joining them back together. The correction to obtain the true least path will be due to the unoptimized connections across the boundaries of the subdivisions. For a suitably made division (not too small), this correction will be on the order of the surface-to-volume ratio of each division, and thus will tend to zero in the $N \rightarrow \infty$ limit. This method, known as "divide and conquer," forms a reasonable strategy for solving approximately such finiterange optimization problems (including finite-range spin glasses) in general. In the second case, the $d_{i j}$ 's are assigned completely randomly, with no geometric (e.g., Euclidean) correlation between them. The problem in this case becomes more like a long-range spin glass. A self-avoiding walk representation of the problem has been made using an $m$-component vector field, and the replica analysis has been done (Mezard et al., 1987) for finite temperature, assuming the replica-symmetry ansatz to hold. Moreover, true breaking of ergodicity may occur only in infinite systems, not in any finite instance of the problem. The results, when extrapolated to zero temperature, do not disagree much with the numerical results (Mezard et al., 1987). The stability of a replicasymmetric solution has not yet been proven for the lowtemperature region. However, the numerical results for thermal annealing, for instance of size $N=60-160$, yielded many near-optimal tours, and the corresponding overlap analysis shows a sharply peaked distribution, whose width decreases steadily with increase in $N$. This indicates the existence of a replica-symmetric phase for the system (Mezard et al., 1987).

An analytical bound on the average (normalized by $N^{1 / 2}$ ) value of the optimal path length per city $(\Omega)$ calculated for the TSP on a two-dimensional Euclidean plane has been found to be $5 / 8<\Omega<0.92$ (Bearwood et al., 1959). Careful scaling analysis of the numerical results obtained indicates the lower bound to be close to 0.72 (Percus and Martin, 1996; Chakraborti and Chakrabarti, 2000).

Simulated (thermal) annealing of a Euclidean TSP on a square having length $N^{1 / 2}$ (which render the average nearest-neighbor distance independent of $N$ ) has been reported (Kirkpatrick et al., 1983). In this choice of length unit, the optimal tour length per step $(\Omega)$ becomes independent of $N$ for large $N$. Thermal annealing 
rendered $\Omega \leq 0.95$ for $N$ up to 6000 cities. This is much better than what is obtained by the so-called greedy heuristics (where, at some city in a step, one moves to the nearest city not in the tour in the next step) for which $\Omega \sim 1.12$ on average. Later we see (Sec. III.A) that quantum annealing can do even better than thermal annealing in the context of the random TSP.

To summarize, when cast as an energy minimization problem, combinatorial optimization problems may exhibit glassy behavior during thermal annealing. Even replica-symmetry-breaking behavior may be observed (as in the case of the graph partitioning problem; see $\mathrm{Fu}$ and Anderson, 1986), since the underlying Hamiltonian need not be short ranged, and the constraints can bring frustration into the problem. One can conclude that thermal annealing or other heuristics would not be able to solve such problems easily to a good approximation within reasonable time. Moreover, almost nothing can be said about the time required to solve the worst case instance exactly. Specifically, in some cases, where good solutions are thermodynamically insignificant in number and there is no monotonic gradient toward them, the entropy might make a classical search exponentially difficult, although the landscape might still remain completely ergodic. Later we see that quantum searches can bring improvements in such cases (see Sec. III.B and Fig. 4).

\section{Quantum spin glasses and annealing}

In QA one adds a kinetic (tunneling) term to the interaction part of the classical glass Hamiltonian. The object that results is called a quantum spin glass. Knowledge of the phase diagram for a quantum spin glass is important for its annealing, as it provides an idea of the location of the quantum critical points on the phase diagram, and thus offers a guideline to choosing the proper kinetic terms (that maintain a sizable gap) and the suitable annealing paths (see Secs. III.A and III.B).

In quantum spin glasses (Chakrabarti, 1981; Ishii and Yamamoto, 1985; Ye et al., 1993; Bhatt, 1998; Sachdev, 1999; Rieger, 2005), the order-disorder transition (i.e., from the frozen phase to the high-kinetic-energy phase, termed the paraphase) can be driven by thermal as well as quantum fluctuations. Quantum spin glasses can be of two types: vector spin glasses, where the quantum fluctuations cannot be adjusted by changing some laboratory field, and classical spin glasses perturbed by some quantum tunneling term, where quantum fluctuations are controlled through, say, a transverse laboratory field.

Since the amount of the quantum fluctuations is adjustable, the transverse Ising spin glass (TISG) model is perhaps the simplest model in which quantum effects in a random system can and have been studied extensively and systematically (Chakrabarti et al., 1996). Here we focus only on the TISG, since reducing quantum fluctuations is the key feature required for quantum annealing.

Interest in the zero-temperature quantum spin-glass phases in TISG models have been complemented all along by experimental studies in several systems that have been shown to be represented by the transverse field Ising model (TIM). The recent discovery that the compound material $\mathrm{LiHo}_{x} \mathrm{Y}_{1-x} \mathrm{~F}_{4}$ with the magnetic Ho ion concentration $x=0.167$ (Wu et al., 1991, 1993; Aeppli and Rosenbaum, 2005; see also Brooke et al., 2001; Silevitch et al., 2007) accurately represents a random longrange transverse Ising system has led to renewed interest. Here the strong spin-orbit coupling between the spins and host crystals restricts the effective "Ising" spins to alignment either parallel or antiparallel to the specific crystal axis. An applied magnetic field, transverse to the preferred axis, flips the Ising spins. This feature, together with the randomness in the spin-spin interaction, makes it a unique TISG-like system. Most interestingly, it has been shown that, in spite of the presence of all three ingredients-frustrations, randomness, and long-range (dipolar) interactions-that are necessary for the formation of a spin glass, the spin-glass phase of $\mathrm{LiHo}_{x} \mathrm{Y}_{1-x} \mathrm{~F}_{4}$ is destroyed by any finite transverse field (Schechter and Laflorencie, 2006). This indicates the effectiveness of quantum tunneling in the exploration of a rugged PEL with formidable potentialenergy barriers. The TISG model described here is given by the Hamiltonian

$$
\mathcal{H}=-\sum_{i>j}^{N} J_{i j} S_{i}^{z} S_{j}^{z}-\Gamma \sum_{i}^{N} S_{i}^{x},
$$

where $\Gamma$ denotes the tunneling strength at each site and the $J_{i j}$ 's are distributed randomly following the distribution $\rho\left(J_{i j}\right)$ given by Eq. (3) or (4). Generally, we denote the strength of the quantum kinetic term by $\Gamma$.

The particular interest in such a quantum spin-glass system comes from the possibility of the much faster crossing of the high barriers occurring in the potentialenergy landscapes of the classical spin glasses by means of quantum tunneling induced by the transverse field, compared to that done thermally by scaling such barriers with the temperature. The phase transitions in quantum spin glasses can be driven by both thermal and quantum fluctuations as mentioned before, and the equilibrium phase diagrams also indicate how the optimized solution (in the SG phase) can be obtained by tuning of either the temperature $T$ or tunneling field $\Gamma$, or both. We show later (in the context of quantum annealing) that reaching the phase by tuning $\Gamma$ may often be more advantageous than by tuning $T$.

The short-range version of this TISG model was first studied by Chakrabarti (1981), and the long-range version, discussed here, was first studied by Ishii and Yamamoto (1985). Several analytical studies have been made to obtain the phase diagram of the transverse Ising SK model (Miller and Huse, 1993). The problem of a SK glass in a transverse field becomes a nontrivial one due to the presence of noncommuting spin operators in the Hamiltonian. This leads to a dynamical frequencydependent self-interaction for the spins.

One can study an effective-spin Hamiltonian for the SK model in a transverse field within the mean-field framework easily. The spin-glass order parameter in a 


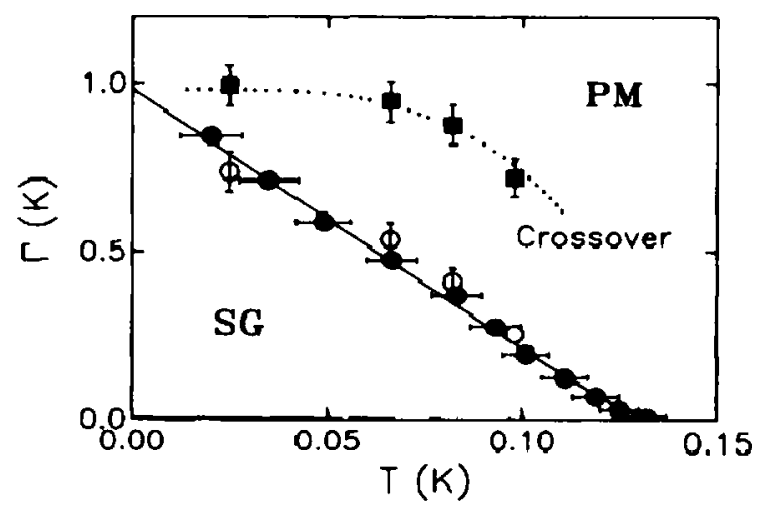

FIG. 2. Phase diagram of $\mathrm{LiHo}_{0.167} \mathrm{Y}_{0.833} \mathrm{~F}_{4}$ according to dynamical (filled circles) and nonlinear susceptibility measurements (open circles). Filled squares indicate the freezing boundary obtained from ac susceptibility measurements. From Wu et al., 1993.

classical SK model is given by a random mean field $h(r)$ having a Gaussian distribution (see Binder and Young, 1986),

$$
q=\int_{-\infty}^{+\infty} d r e^{-r^{2} / 2} \tanh ^{2}\left[h^{z}(r) / T\right], \quad h^{z}(r)=J \sqrt{q} r+h^{z},
$$

where $h^{z}$ denotes the external field (in the $z$ direction), with the mean field $h(r)$ also in the same direction. In the presence of the transverse field, as in Eq. (9), $h(r)$ has components in both the $z$ and $x$ directions,

$$
\vec{h}(r)=-h^{z}(r) \hat{z}-\Gamma \hat{x}, \quad h(r)=\sqrt{h^{z}(r)^{2}+\Gamma^{2}},
$$

and one replaces the ordering term $\tanh ^{2}[h(r) / T]$ in Eq. (10) by its component $\left[\left|h^{z}(r)\right| /|h(r)|\right]^{2} \tanh ^{2}[|h(r)| / T]$ in the $z$ direction. Setting $h^{z}=0$ and $q \rightarrow 0$, one gets the phase boundary equation as (see Chakrabarti et al., 1996)

$$
\frac{\Gamma}{J}=\tanh \left(\frac{\Gamma}{T}\right) .
$$

This gives $\Gamma_{c}(T=0)=J=T_{c}(\Gamma=0)$ and a phase diagram qualitatively similar to the experimental one shown in Fig. 2.

Several Monte Carlo studies have been performed for the SK spin-glass model in the transverse field by applying the Suzuki-Trotter formalism (see Appendix A.1), mapping a $d$-dimensional quantum Hamiltonian to an effective $(d+1)$-dimensional anisotropic classical Hamiltonian (see also Hatano and Suzuki, 2005). The partition function gives the effective classical Hamiltonian in the $M$ th Trotter approximation as

$$
\mathcal{H}=\sum_{i>j}^{N} \sum_{k}^{M} K_{i j} S_{i k} S_{j k}-\sum_{i}^{N} \sum_{k}^{M} K S_{i k} S_{i k+1}
$$

with

$$
K_{i j}=\frac{J_{i j}}{M T}, \quad K=\frac{1}{2} \ln \operatorname{coth}\left(\frac{\Gamma}{M T}\right),
$$

where $S_{i k}$ denotes the Ising spin defined on the lattice site $(i, k), i$ denoting the position in the original SK model and $k$ that in the additional Trotter dimension. Although the equivalence between classical and quantum models holds exactly in the limit $M \rightarrow \infty$, one can always make an optimum choice for $M$. The equivalent classical Hamiltonian has been studied using the standard Monte Carlo technique. Numerical estimates of the phase diagram, etc., have been reviewed by Bhatt (1998) and Rieger (2005). Ray et al. (1989) took $\Gamma \ll J$, and their results indicated a sharp lowering of $T_{c}(\Gamma)$. Such a sharp fall of $T_{c}(\Gamma)$ with large $\Gamma$ is obtained in almost all theoretical studies of the phase diagram of the model (Miller and Huse, 1993; Ye et al., 1993; see also Bhatt, 1998, and Rieger, 2005). Quantum Monte Carlo (Alvarez and Ritort, 1996) as well as real-time Schrödinger evolution (the true dynamics given by the time-dependent Schrödinger equation) studies of SK spin glasses in transverse field have been made (Lancaster and Ritort, 1997).

In the Hamiltonian for the EA spin glass in the presence of a transverse field, given by Eq. (9), the random interactions are restricted to the nearest neighbors and satisfy a Gaussian distribution with zero mean and variance $J$, as given by Eq. (3). Here the variation of correlations in the equivalent $(d+1)$-dimensional classical model agreed well (Guo et al., 1994) with the scaling fit with a unique order parameter and a critical interval corresponding to a phase diagram whose features are similar to those discussed above (see also Chakrabarti et al., 1996; Bhatt, 1998; Rieger, 2005).

As discussed earlier in this section, $\mathrm{LiHo}_{x} \mathrm{Y}_{1-x} \mathrm{~F}_{4}$ with $x=0.167$ provides a spin-glass system for which the external magnetic field transverse to the preferred axis scales as the square root of the tunneling field $\Gamma$ in Eq. (9). With increasing transverse field, the glass transition temperature decreases monotonically, as shown in Fig. 2.

A quantum tunneling term allows for overlap between two classically localized states and the dynamics near the ground states of such glasses show better ergodicity properties. In order to investigate this aspect of quantum spin glasses, one can study the overlap distribution function $P(q)$ given by Eq. (6).

If the ergodicity is recovered, at least for a part of the phase diagram, the above function should tend to a delta function form, peaking at some finite value of the order parameter $q$ in the thermodynamic limit. In the paraphase the distribution becomes a delta function at $q=0$ for the infinite system. In spite of several investigations (see, e.g., Ray et al., 1989; Thirumalai and Kirkpatrick, 1989; Goldschmidt and Lai, 1990; Chakrabarti et al., 1996; Kim and Kim, 2002) the question of replicasymmetry restoration in spin glasses by quantum fluctuations is not settled yet. However, slow withdrawal [see Eq. (16) for the characteristic slowness] of the tunneling field in these quantum spin glasses can help in 
annealing the system close to the ground state of the classical spin glass eventually, as described in the next section.

\section{QUANTUM ANNEALING}

In the previous sections we have seen how thermal fluctuations can be utilized to devise fast heuristics to find an approximate ground state of a glassy system, or equivalently a near-optimal solution to the combinatorial problem, whose cost-configuration landscape has glassy behavior due to the occurrence of many local minima. There are two aspects of an optimization problem which might render thermal annealing an ineffective one. First, in a glassy landscape, there may exist highcost or -energy barriers around local minima which do not correspond to a reasonably low cost (see Fig. 1). In the case of infinite-range problems, these barriers might be proportional to the system size $N$, and thus diverge in thermodynamic limit. Thus many unsatisfactory local minima might occur, any of which can trap the system for a long time (which actually diverges in the thermodynamic limit for infinite-range systems) in the course of annealing. The second problem is the entropy itself. The number of configurations grows fast with the number of variables (roughly exponentially; $n$ Ising spins can be in $2^{n}$ configurations), and a classical system can assume only one configuration at a time; unless there is a gradient that broadly guides the system toward the global minimum from any point in the configuration space, the search has to involve visiting a substantial fraction of configurations. Thus a PEL without a guiding gradient poses a problem which is exponential or higher order in complexity (depending on how the size of the configuration space scales with the system size $N$ ), and the CA algorithms can do no better than a random search algorithm. This is the case for the golf-course-type potentialenergy landscape, where there is a sharp potential minimum on a completely flat PEL (see Sec. III.B, Fig. 4). One can imagine that quantum mechanics might have solutions to both these problems, at least to some extent. This is because quantum mechanics can introduce classically unlikely tunneling paths even through very high barriers if they are narrow enough (Ray et al., 1989; see also Apolloni et al., 1989, 1990). This can solve the ergodicity problem to some extent, as discussed earlier. Even in places where ergodicity breaking does not take place in the true sense, once the energy landscape contains high enough barriers (especially for infinite-ranged quenched interactions), quantum tunneling may provide much faster relaxation to the ground state (Martońák et al., 2002; Santoro et al., 2002; see also Santoro and Tosatti, 2007; Somma et al., 2007). In addition, a quantum-mechanical wave function can delocalize over the whole configuration space (i.e., over the potentialenergy landscape) if the kinetic energy term is high enough. Thus it can actually "see" the whole landscape simultaneously at some stage of annealing. These two aspects can be expected to improve the search process when employed properly. In fact, such improvements can be achieved in certain situations, though quantum mechanics is not a panacea for all such problems as ergodicity breaking, spin-glass behavior, etc., and certainly has its own inherent limitations. What is intriguing is the fact that the limitations due to the quantum nature of an algorithm are inherently different from those faced by its classical counterpart, and thus it is not yet clear in general which is better when. Here we discuss results regarding quantum heuristics and some of their general aspects that are understood so far. For more detailed reviews of the subject we refer the reader to articles by Das and Chakrabarti (2005) and Santoro and Tosatti (2006).

Some basic aspects of QA can be understood from the simple case of QA in the context of a double-well potential (Battaglia, Stella, et al., 2005; Stella et al., 2005). Typically a particle in a double well consisting of a shallower but wider well and a deeper but narrower well is annealed (it is likely that the deeper well, i.e., the target state, is narrower, otherwise searching becomes easier even classically). The kinetic energy (inverse mass) is tuned from a very high value to zero linearly within a time $\tau$. For a very high value of initial kinetic energy, the wave function, which is the ground state, is delocalized more or less over the whole double well. As kinetic energy is reduced but still quite high, the ground state corresponds to a more pronounced peak on the shallower minimum, since it is wider. This is because at this stage, to obtain the minimal (ground-state) energy, it is more effective to minimize the kinetic energy by localization over a wider space, rather than minimizing the potential energy by localizing in the deeper well. However, as the kinetic energy is reduced further, the potential energy term dominates, and the ground state has a taller peak around the deeper minimum. The evolving wave function can roughly follow this ground-state structure all the way and finally settle to the deeper minimum if the annealing time $\tau$ is greater than $\tau_{c}$. When $\tau<\tau_{c}$ the wave function fails to tunnel from its early state localized in the shallower well to the deeper well as the kinetic energy is decreased. This result is qualitatively the same for both real-time and quantum Monte Carlo annealing, except for the fact that the $\tau_{c}$ 's are different in the two cases.

The realization of QA consists of employing adjustable quantum fluctuations into the problem instead of a thermal one (Amara et al., 1993; Finnila et al., 1994; Kadowaki and Nishimori, 1998). In order to do that, one needs to introduce an artificial quantum kinetic term $\Gamma(t) \mathcal{H}_{\text {kin }}$, which does not commute with the classical Hamiltonian $\mathcal{H}_{C}$ representing the cost function. The coefficient $\Gamma$ is the parameter that controls the quantum fluctuations. The total Hamiltonian is thus given by

$$
\mathcal{H}_{\text {tot }}=\mathcal{H}_{C}+\Gamma(t) \mathcal{H}_{\text {kin }}
$$

The ground state of $\mathcal{H}_{\text {tot }}$ is a superposition of the eigenstates of $H_{C}$. For a classical Ising Hamiltonian of the form (2), the corresponding total quantum Hamiltonian might have the form (9), where $\mathcal{H}_{C}=-\Sigma_{i>j} S_{i}^{z} S_{j}^{z}$ and 
$\mathcal{H}_{\text {kin }}=-\sum_{i}^{N} S_{i}^{x}$. Initially $\Gamma$ is kept high so that $\mathcal{H}_{\text {kin }}$ dominates and the ground state is trivially a uniform superposition of all classical configurations. One starts with that uniform superposition as the initial state, and slowly decreases $\Gamma$ following some annealing schedule, eventually to zero. If the process of decreasing is slow enough, the adiabatic theorem of quantum mechanics (Sarandy et al. , 2004) assures that the system will always remain at the instantaneous ground state of the evolving Hamiltonian $\mathcal{H}_{\text {tot }}$. When $\Gamma$ is finally brought to zero, $\mathcal{H}_{\text {tot }}$ will coincide with the original classical Hamiltonian $\mathcal{H}_{C}$ and the system will be found in its ground state, as desired. The special class of QA algorithms where strictly quasistationary or adiabatic evolutions are employed are also known as quantum adiabatic evolution algorithms (Farhi, Goldstone, Gutmann, et al., 2000, 2001).

Two important questions are how to choose an appropriate $\mathcal{H}_{\text {kin }}$ and how slow the evolution needs to be in order to assure adiabaticity. According to the adiabatic theorem of quantum mechanics, for a nondegenerate spectrum with a gap between the ground state and first excited state, adiabatic evolution is assured if the evolution time $\tau$ satisfies the following condition:

$$
\tau \gg \frac{\left|\left\langle\dot{\mathcal{H}}_{\text {tot }}\right\rangle\right|_{\max }}{\Delta_{\min }^{2}},
$$

where

$$
\begin{aligned}
& \left|\left\langle\dot{\mathcal{H}}_{\text {tot }}\right\rangle\right|_{\max }=\max _{0 \leq t \leq \tau}\left(\left|\left\langle\phi_{0}(t)\left|\frac{d \mathcal{H}_{\text {tot }}}{d s}\right| \phi_{1}(t)\right\rangle\right|\right), \\
& \Delta_{\min }^{2}=\min _{0 \leq t \leq \tau}\left[\Delta^{2}(t)\right], \quad s=t / \tau, \quad 0 \leq s \leq 1,
\end{aligned}
$$

$\left|\phi_{0}(t)\right\rangle$ and $\left|\phi_{1}(t)\right\rangle$ are, respectively, the instantaneous ground state and the first excited state of the total Hamiltonian $\mathcal{H}_{\text {tot }}$, and $\Delta(t)$ is the instantaneous gap between the ground-state and first excited-state energies (see Sarandy et al., 2004). One may wonder whether, on entering the ordered phase $\left(\Gamma<\Gamma_{c}\right)$ from the paraphase $\left(\Gamma>\Gamma_{c}\right)$ in the course of annealing, the gap $\Delta$ may vanish at the phase boundary $\left(\Gamma=\Gamma_{c}\right)$ in the $N \rightarrow \infty$ limit. In fact, in such a case, QA cannot help in finding the ground state of an infinite system. However, for any finite sample, this gap is unlikely to vanish for a random system, and QA may still work.

However, it is impossible to follow, even for finite $N$, the evolution of a full wave function in a classical computer using polynomial resources in general, since it requires tracking the amplitudes of all basis vectors (all possible classical configurations), whose number grows exponentially with system size $N$. Such an adiabatic evolution may be realized within polynomial resources only if one can employ a quantum-mechanical system itself to mimic the dynamics. However, one may employ quantum Monte Carlo methods to simulate some dynamics (not the real-time quantum dynamics) to sample the ground state (or a mixed state at low enough temperature) for a given set of parameter values of the Hamiltonian. Annealing is done by reducing the strength $\Gamma$ of the quantum kinetic term in the Hamiltonian from a very high value to zero following some annealing schedule in the course of simulation. In the case of such a Monte Carlo annealing algorithm, there is no general bound on success time $\tau$ such as the one provided by the adiabatic theorem for true Schrödinger evolution annealing. Here we separately discuss the results of realtime QA and Monte Carlo QA. Apart from these quasistationary quantum annealing strategies, where the system always stays close to some stationary state (or low-temperature equilibrium state), there may be cases where quantum scatterings (with tunable amplitudes) are employed to anneal the system (Das et al., 2005).

\section{A. Quantum Monte Carlo annealing}

In quantum Monte Carlo annealing, one may employ either a finite- (but low-) temperature algorithm or a zero-temperature algorithm. Most Monte Carlo QAs (Das and Chakrabarti, 2005; Santoro and Tosatti, 2006) are done using a finite-temperature Monte Carlo method, namely, the path-integral Monte Carlo (PIMC), since its implementation is somewhat simpler than that of other zero-temperature Monte Carlo methods.

Among the other zero-temperature Monte Carlo methods used for annealing are the zero-temperature transfer-matrix Monte Carlo (see the chapter by Das and Chakrabarti in Das and Chakrabarti, 2005) and the Green's function Monte Carlo (Santoro and Tosatti, 2006) methods. However, these algorithms suffer severely from different drawbacks, which renders them much slower than PIMC algorithms in practice.

The Green's function Monte Carlo algorithm effectively simulates the real-time evolution of the wave function during annealing. But to perform the algorithms sensibly often requires guidance that depends on a priori knowledge of the wave function. Without this guidance it may fail miserably (Santoro and Tosatti, 2006). But such a priori knowledge is unlikely to be available in the case of random optimization problems, and hence so far the scope for this algorithm seems to be very restricted.

The zero-temperature transfer-matrix Monte Carlo method, on the other hand, samples the ground state of the instantaneous Hamiltonian (specified by the given value of the parameters at that instant) using a projective method, where the Hamiltonian matrix itself (a suitable linear transformation of the Hamiltonian that converts into a positive matrix, in practice) is viewed as the transfer matrix of a finite-temperature classical system of one higher dimension (Das and Chakrabarti, 2005). But the sparsity of the Hamiltonian matrix for systems with local kinetic energy terms leaves the classical system highly constrained and thus difficult to simulate efficiently for large system sizes.

The PIMC algorithm has so far been mostly used for QA. The basic idea of this method rests on the SuzukiTrotter formalism (see Appendix A.1), which maps the partition function of a $d$-dimensional quantum Hamiltonian $\mathcal{H}$ onto that of an effective classical Hamiltonian 
$\mathcal{H}_{\text {eff }}$ in $d+1$ dimensions. Quantum annealing of a Hamiltonian $\mathcal{H}_{\text {tot }}$ using the PIMC method consists of mapping $\mathcal{H}_{\text {tot }}$ to its equivalent classical one and simulate it at some fixed low temperature so that thermal fluctuations are low. Quantum fluctuations are reduced from some very high initial value to zero finally through reduction of $\Gamma$ in the course of simulation. Clearly, the simulation dynamics is not the true Schrödinger evolution of the system, and also it cannot simultaneously see the whole configuration space as does a delocalized wave function. It experiences the landscape locally and makes moves as in a classical system. The first attempt at quantum annealing using the PIMC method was made by Kadowaki and Nishimori (1998) for solving the TSP, and extensive use of the technique to explore a multitude of problems has been made by Santoro and Tosatti (2006). Here we discuss some PIMC quantum annealing results for a few different systems.

\section{A short-range spin glass}

Quantum annealing of an EA spin glass in two dimensions (square lattice) using a transverse field [see Eq. (9)] for large lattice size (up to $80 \times 80$ ) using the PIMC technique turns out to be much more efficient compared to thermal annealing (CA) of the same system in finding the approximate ground state (Martonák et al., 2002; Santoro et al., 2002). The quantity that is measured is the residual energy $\epsilon_{\mathrm{res}}(\tau)=E(\tau)-E_{0}$, with $E_{0}$ the true ground-state energy of the finite system ${ }^{1}$ and $E(\tau)$ the final energy of the system after reducing the transverse field strength $\Gamma$ linearly with time $\tau$ from some large initial value to zero. Here $\tau$ is a fictitious time given by the number of Monte Carlo steps.

Classically, for a large class of frustrated disordered system it can be shown, using general arguments, that the residual energy decreases following some power law in the logarithm of the annealing time $\tau$, namely, $\epsilon_{\text {res }}$ $\sim(\ln \tau)^{-\zeta}$, with $\zeta \leq 2$ (Huse and Fisher, 1986). In PIMC simulations, the partition function of a $d$-dimensional quantum system is mapped to an equivalent $(d+1)$ dimensional classical system (this is known as SuzukiTrotter mapping). The effective $(d+1)$-dimensional system is obtained by replicating the classical part of the original system (say, the Ising interaction part of the transverse Ising system without the transverse field term) with all its interactions (including the disorder) intact along the extra higher dimension. The coupling between the spins in different replicas depends on the quantum kinetic term in the original $d$-dimensional system.

The PIMC annealing results show, however, that the quantum effect (taken into account through SuzukiTrotter mapping) does not change the relaxation behavior of $\epsilon_{\text {res }}(\tau)$. But a dramatic improvement in evaluation

\footnotetext{
${ }^{1}$ Calculated by the spin-glass server using the so-called branch-and-cut algorithms; see http://www.informatik.unikoeln.de/ls juenger/research/spinglass/.
}

time is still achieved, since it turns out that the value of the exponent $\zeta$ can be much higher $(\zeta=6)$ for QA than the Huse-Fisher bound of $\zeta \leq 2$ for classical annealing. This is an improvement in computational time if one thinks in terms of the changes in $\tau$ required to improve $\epsilon_{\text {res }}$ equally by some appreciable factor in the corresponding cases of classical and quantum annealing. An interesting asymptotic comparison for the results of QA and $\mathrm{CA}$ for an $80 \times 80$ lattice shows that, to reach a certain value of $\epsilon_{\text {res }}$, PIMC QA would take one day of CPU time (for the computer used) whereas CA would take about 30 years (Martoňák et al., 2002; Santoro et al., 2002). The result would not be much different in this case if the real-time Schrödinger evolution were followed, as has been argued using the Landau-Zener cascade tunneling picture (Martoňák et al., 2002; Santoro et al., 2002).

Landau-Zener tunneling theory gives as an estimate the probability of tunneling nonadiabatically from a lower to a higher level when the system encounters an avoided level crossing between levels during time evolution. Let the gap between two energy levels $|a(t)\rangle$ and $|b(t)\rangle$ of a time-dependent Hamiltonian vary linearly with time (gap $\Delta=\alpha t)$ and encounter an avoided level crossing. Here the levels are energy levels of the classical part of the Hamiltonian (say, the potential-energy levels of an Ising model). Let the system evolve in such a way that the characteristic time it spends while passing through the crossing region is $\tau$. Let there be a quantum tunneling field $\Gamma$ that induces transitions between levels. Then if the system evolved is at the lower branch $|a\rangle$ before encountering the avoided level crossing, the probability that it continues to the higher branch $|b\rangle$ while passing through the crossing decreases with the time $\tau$ as $P(\tau)=\exp \left(-\tau / \tau_{\Gamma}\right)$, where $\tau_{\Gamma}=\hbar \alpha \Gamma / 2 \pi \Delta_{\min }^{2}$, with $\Delta_{\text {min }}$ the minimum value of the gap attained at the avoided level crossing. For spin-glass-like systems with nonzero gap, by treating multiple level crossings, each with small $\Delta$, as a cascade of independent Landau-Zener tunneling one may argue that the residual energy varies as $\epsilon_{\text {res }} \sim(\ln \tau)^{-\zeta_{Q}}$, where $\zeta_{Q}$ is greater than the bound $\zeta$ $\leq 2$ for thermal annealing, and might be as high as 6 (Martoňák et al., 2002; Santoro et al., 2002).

\section{The traveling salesman problem}

Quantum annealing of the TSP with a random metric (i.e., the distance $d_{i j}$ between $i$ th and the $j$ th cities is chosen randomly) in infinite dimension using the PIMC algorithm was also found to be more efficient than CA in finding an approximately minimal tour (Martoňák et al., 2004). An $N$-city tour in a random TSP can be represented by a configuration of $N^{2}$ constrained Ising spins, and the tour length, i.e., $\mathcal{H}_{c}$, by the Hamiltonian (8). Now, to do the annealing, one needs to introduce a set of moves (spin-flip operations) that satisfies the constraints. Classically, one important class of moves is the two-opt moves, which, starting from a valid tour, can generate all possible tours without generating any in- 


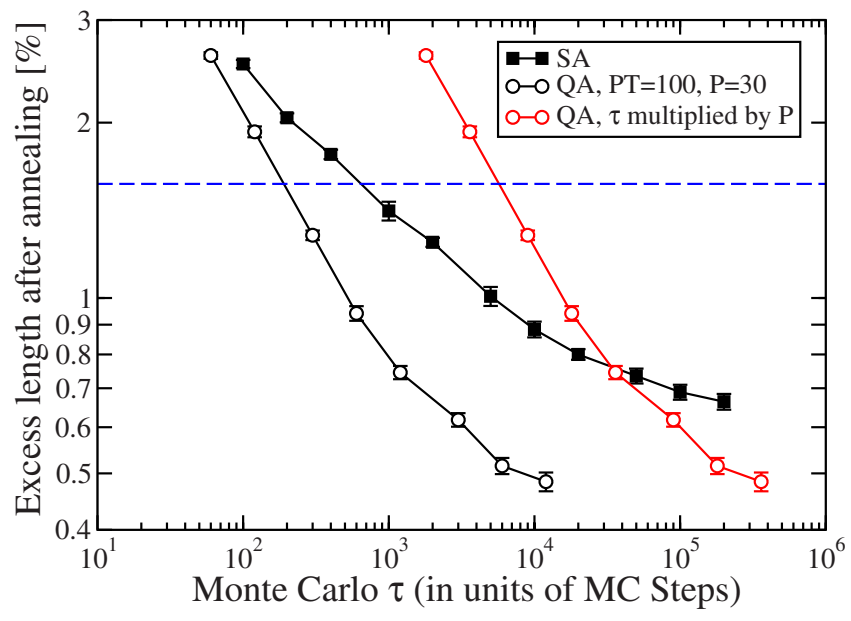

FIG. 3. (Color online) Average residual excess length found after CA and QA for a total time $\tau$ (in MC steps), for the $N$ $=1002$ instance pr1002 of the TSPLIB. The dashed horizontal line represents the best out of 1000 runs of the Lin-Kernighan algorithm. QA is clearly faster than CA. From Battaglia, Stella, et al., 2005.

valid link. Let a valid tour contain two links $i \rightarrow j$ and $k \rightarrow l$. A two-opt move may consist of removing those two links and establishing the following links: $i \rightarrow k$ and $j \rightarrow l$ (here $i$ denotes the $i$ th city). Classical annealing of the Hamiltonian can be done by restricting the Monte Carlo moves within the two-opt family only. However, for the quantum case, one needs to design a special transverse field (noncommuting spin-flip term) to enforce the constraints. The constraint can be realized by a spin-flip term of the form $S_{\langle k, i\rangle}^{+} S_{\langle l, j\rangle}^{+} S_{\langle j, i\rangle}^{-} S_{\langle l, k\rangle}^{-}$, where the operator $S_{i, j}^{-}$flips down $(+1 \rightarrow-1)$ the Ising spins $S_{i j}^{z}$ and $S_{j i}^{z}$ when they are in the +1 state, and similarly for the flip-up operators $S_{i, j}^{+}$. However, to avoid the SuzukiTrotter mapping with these complicated kinetic terms, a relatively simple kinetic term of the form $\mathcal{H}_{\text {kin }}$ $=-\Gamma(t) \sum_{\langle i, j\rangle}\left(S_{\langle i, j\rangle}^{+}+\right.$H.c. $)$is used for the quantum to classical mapping. The Monte Carlo moves were restricted within the two-opt family to avoid invalid tours. The results were tested on the instance of a printed circuit board with $N=1002$. PIMC QA was seen to do better than the CA and also much better than the standard Lin-Kernighan algorithm, as shown in Fig. 3. The relaxation behavior of the residual path length for the TSP (see Fig. 3) is found to be quite similar to that of the 2D EA glass discussed earlier (see Battaglia, Stella, et al., 2005). This indicates that a random TSP also has a spinglass-like potential-energy landscape, as has already been hinted by the replica-analysis study of the problem discussed earlier (Sec. II.C.2). In that case the LandauZener tunneling picture would also be applicable for the TSP, and little improvement can be expected by following the real-time Schrödinger dynamics instead of Monte Carlo methods.

There are cases where PIMC QA is not as successful as CA. An example (discussed below) of such a problem belongs to the class of $K$-satisfiability problem (or
$K$-SAT). In a $K$-SAT problem, there is a given Boolean function, which is the Boolean sum (connected by logical OR operations) of a number of clauses, each of which is a Boolean product (connected by logical AND operations) of $K$ Boolean variables (binary variables taking values 0 or 1 only) taken randomly from a given set of $p$ variables (the same variable may occur in various clauses simultaneously). Given such a function, the task is to find an assignment for the Boolean variables for which the number of violated clauses (the clauses assigned the 0 value) is minimum. Studies on this class of problem are extensive and even include the connection between its hardness and satisfiability-unsatisfiability phase transition (Monsasson et al., 1999). Remarkable progress has been made in formulating faster algorithms for solving it (see Mezard et al., 1987; Hartmann and Rieger, 2002) based on these understandings.

But in the case of the random 3-SAT problem ( $K$-SAT problem with $K=3$ ) using a linear schedule for decreasing $\Gamma$, PIMC QA gives much worse results than CA (Battaglia, Santoro, and Tosatti, 2005). Both CA and PIMC QA are worse than ad hoc local search heuristics like WALKSAT. In applying the PIMC QA in image restoration, on the other hand, the performance is exactly the same as that of CA (Inoue, 2005). Even for a particle in a simple double-well potential, it seems that with naive Monte Carlo moves, PIMC QA can produce results that are much worse than one could obtain from realtime Schrödinger evolution of the system. There is, in fact, no general prescription for choosing the right moves that will work, unless one has a precise idea about the PEL of the problem. The choice of the kinetic term to be introduced into the problem is somewhat arbitrary, but the performance of the algorithm depends crucially on it. It has been found that a relativistic kinetic term can do a better job than a nonrelativistic one in the case of a particle in a double-well potential (Battaglia, Stella, et al., 2005). PIMC QA also suffers from difficulty in calculating the Suzuki-Trotter equivalent of the quantum Hamiltonian with an arbitrary kinetic term designed to satisfy the constraints of the problem (Martonák et al., 2004). The constraints may be taken care of while making Monte Carlo moves, but that may not always produce the expected results. Finally, the presence of finite temperature in the problem does not allow one to focus exclusively on the role of quantum fluctuations in the problem. Above all, like any other Monte Carlo method, PIMC QA is going to do worse if there are only a few reasonably good approximate solutions, and there is no overall gradient in the landscape to guide toward them.

\section{Random field Ising model: How the choice of kinetic term improves annealing results}

QA algorithms enjoy an extra flexibility which is not available to a thermal annealing algorithm. A QA algorithm can have a host of choices for its kinetic term. A good choice can bring about much improvements. This point has been illustrated by Suzuki et al. (2007) for QA 
of the random field Ising model, by introducing a ferromagnetic transverse field interaction, in addition to the conventional single-spin-flip transverse field term [as present in the Hamiltonian (9)].

The Hamiltonian of the random-field Ising model with the standard single-spin-flip transverse term is given by

$$
\mathcal{H}(t)=\mathcal{H}_{C}+\mathcal{H}_{\text {kin }}^{(1)},
$$

where

$$
\mathcal{H}_{C}=-J \sum_{\langle i j\rangle}^{N} S_{i}^{z} S_{j}^{z}-\sum_{i=1}^{N} h_{i}^{z} S_{i}^{z},
$$

$h_{i}^{z}$ is the on-site random field, assuming values +1 or -1 with equal probabilities, $\langle i j\rangle$ denotes the sum over nearest neighbors on a two-dimensional square lattice, and

$$
\mathcal{H}_{\mathrm{kin}}^{(1)}=-\Gamma(t) \sum_{i=1}^{n} S_{i}^{x} .
$$

The result of QA in such a system is not satisfactory when $J$ is much larger than $h_{i}^{z}$ (Sarjala et al., 2006). If a ferromagnetic transverse term of the form

$$
\mathcal{H}_{\text {kin }}^{(2)}=-\Gamma(t) \sum_{\langle i j\rangle}^{N} S_{i}^{x} S_{j}^{x}
$$

is added to the Hamiltonian (18), the result of QA is seen to improve considerably (Suzuki et al., 2007). This happens (as indicated by exact diagonalization results on small systems) because the ferromagnetic transverse field term effectively increases the gap between the ground state and the first excited state and thus decreases the characteristic time scale for the system. This is an example of how one can utilize the flexibility in choosing the kinetic term in QA to formulate faster algorithms. This also indicates how knowledge of the system's phase diagram, the position of the quantum critical point in particular (where the gap tends to vanish), helps in choosing additional kinetic terms and thus allows for finding annealing paths that can avoid, to some extent, the regions of very low gap.

\section{B. Quantum annealing using real-time adiabatic evolution}

QA is basically the analog version of quantum computation. As for conventional analog quantum computation, the hardware realization of adiabatic quantum annealing is rather problem specific. But once realized, it follows the real-time Schrödinger evolution, whose exact simulation is always intractable (the run time grows exponentially with the system size) for classical computers and often also even for digital quantum computers (see Nielsen and Chuang, 2000). The annealing behavior with the real-time Schrödinger evolution is hence an important issue and may show features distinctly different from any Monte Carlo annealing discussed so far.

The first analog algorithm was formulated by Farhi and Gutmann (1998) for solving Grover's search problem. Grover (1997) showed that a quantum-mechanical search can reduce an $O(N)$ classical search time to $O(\sqrt{N})$ in finding a marked item from an unstructured database. In the analog version, the problem was to use quantum evolution to find a given marked state among $N$ orthonormal states. The algorithm was formulated in the following way. There were $N$ mutually orthogonal normalized states, the $i$ th state denoted by $|i\rangle$. Among all of them, only one, say, the $w$ th one, has energy $E \neq 0$ and the rest all have zero energy. Thus the state $|w\rangle$ is "marked" energetically and the system can distinguish it. Now the question is how fast the system can evolve under a certain Hamiltonian so that starting from an equal superposition of the $N$ states one reaches $|w\rangle$. It was shown (Farhi and Gutmann, 1998) that if the system evolves under a time-independent Hamiltonian of the form

$$
\mathcal{H}_{\text {tot }}=E|w\rangle\langle w|+E| s\rangle\langle s|,
$$

where

$$
|s\rangle=\frac{1}{\sqrt{N}} \sum_{i=1}^{N}|i\rangle,
$$

no improvement over Grover's $\sqrt{N}$ speedup is possible. Later, the problem was recast in the form of a spatial search (Childs and Goldstone, 2004), where there is a $d$-dimensional lattice and the basis state $|i\rangle$ is localized at the $i$ th lattice site. As before, the on-site potential energy $E$ is zero everywhere except at $|w\rangle$, where it is 1 . The objective is to reach the marked state starting from the equal superposition of all the $|i\rangle$ 's. The kinetic term is formulated through the Laplacian of the lattice, which effectively introduces uniform hopping to all nearest neighbors from any given lattice sites, and is kept constant. The model is in essence an Anderson model (Santoro and Tosatti, 2006) with only a single-site disorder of strength $O(1)$. Grover's speedup can be achieved for $d$ $\geq 4$ with such a Hamiltonian and no further improvement is possible. The algorithm succeeds only near the critical value of the kinetic term (i.e., at the quantum phase transition point). An adiabatic quantum evolution algorithm for Grover search was formulated in terms of an orthonormal complete set of $l$-bit Ising-like basis vectors, where the potential energy of a given basis vector (among $2^{l}$ ) is 1 , and for the rest it is 0 . The kinetic term is the sum of all single-bit-flip terms [like the transverse field term in Eq. (9)]. It connects each basis vector to those that can be reached from it by a single-bit flip. The kinetic term is reduced from a high value to zero following a linear schedule. A detailed analysis in light of the adiabatic theorem showed that one cannot even retrieve Grover's speedup by sticking to the global adiabatic condition with fixed evolution rate as given by Eq. (16). This occurs because the minimum value of the gap varies as $\Delta_{\min } \sim 2.2^{-l / 2}$ (Farhi et al., 2000), and since the spin-flip kinetic term is local, the numerator $\left|\left\langle\dot{\mathcal{H}}_{\text {tot }}\right\rangle\right|$ of the adiabatic factor [see Eq. (16)] is at best $O(l)$. However, Grover's speedup can be recovered if the condition of adiabaticity is maintained locally [for more on nonlinear 
annealing schedule, see also Farhi et al. (2002b) and Morita (2007)] at every instant of the evolution and the rate is accelerated accordingly whenever possible (Roland and Cerf, 2001). Adiabatic QA following real-time Schrödinger evolution for satisfiability problems also gives a different result (for smaller system size, though) compared to that obtained using PIMC QA. Adiabatic $\mathrm{QA}$ of an NP-complete problem, namely, the exact cover problem (as described below) has been studied for small systems, where a quadratic system-size dependence was observed (Farhi, Goldstone, Gutmann, et al., 2001). In this problem the basis vectors are the complete set of $2^{l}$ orthonormal $l$-bit basis vectors, denoted by $\left.\left\{\left|z_{1}\right\rangle|| z_{2}\right\rangle \cdots\left|z_{l}\right\rangle\right\}$; each $z_{i}$ is either 0 or 1 . The problem consists of a cost function $\mathcal{H}_{C}$ which is the sum of many three-bit clause functions $h_{\mathrm{Cl}}\left(z_{i}, z_{j}, z_{k}\right)$, each acting on arbitrarily chosen bits $z_{i}, z_{j}$, and $z_{k}$. The clause function is such that $h_{\mathrm{Cl}}\left(z_{i}, z_{j}, z_{k}\right)=0$ if the clause $\mathrm{Cl}$ is satisfied by the states $\left|z_{i}\right\rangle,\left|z_{j}\right\rangle$, and $\left|z_{k}\right\rangle$ of the three bits, or else $h_{\mathrm{Cl}}\left(z_{i}, z_{j}, z_{k}\right)=1$. The cost Hamiltonian is given by $\mathcal{H}_{C}$ $=\Sigma_{\mathrm{Cl}} h_{\mathrm{Cl}}$. Thus if a basis state $\left|\left\{z_{i}\right\}\right\rangle$ dissatisfies $p$ clauses, then $\mathcal{H}_{C}\left|\left\{z_{i}\right\}\right\rangle=p\left|\left\{z_{i}\right\}\right\rangle$. The question is whether there exists a basis vector that satisfies all clauses for a given $\mathcal{H}_{C}$. There may be many basis vectors satisfying a clause. All the basis vectors will be the ground state of $H_{C}$ with zero eigenvalue. If the ground state has a nonzero eigenvalue (it must be a positive integer then) then it represents the basis with the lowest number of violated clauses, the number given by the eigenvalue itself. The total Hamiltonian is given by

$$
\mathcal{H}_{\text {tot }}(t)=\left(1-\frac{t}{\tau}\right) \mathcal{H}_{\text {kin }}+\frac{t}{\tau} \mathcal{H}_{C}
$$

where $\mathcal{H}_{\text {kin }}$ is again the sum of all single-bit-flip operators. The initial state at $t=0$ is taken to be the ground state of $\mathcal{H}_{\text {kin }}$, which is an equal superposition of all basis vectors. The system then evolves according to the timedependent Schrödinger equation up to $t=\tau$. The values of $\tau$ required to achieve a preassigned success probability are noted for different system sizes. The result showed a smooth quadratic system-size dependence for $l \leq 20$ (Farhi, Goldstone, and Gutmann, 2002b). which is quite encouraging (since it seems to give a polynomial time solution for an NP-complete problem for small system size), but does not really assure a quadratic behavior in the asymptotic $(l \rightarrow \infty)$ limit.

Quadratic relaxation behavior $\left(\epsilon_{\mathrm{res}} \sim 1 / \tau^{\zeta}, \zeta \sim 2\right)$ was reported by Suzuki and Okada (2005a) for real-time adiabatic QA [employing the exact method for small systems and the density-matrix renormalization group (DMRG) technique for larger systems (see Suzuki and Okada, 2005b)] of a one-dimensional tight-binding model with random site energies and also for a randomfield Ising model on a 2D square lattice. In the first case, the kinetic term was due to hopping between nearest neighbors, while in the second case it was simply the sum of single-spin-flip operators as given by Eq. (9). Wave-function annealing results using a similar DMRG technique for a spin glass on a ladder have been reported by Rodriguez-Laguna (2007).

It has been demonstrated that for finite-ranged systems, where the interaction energy can be written as a sum of interaction energies involving few variables, quantum adiabatic annealing and thermal annealing may not differ much in efficiency. However, for problems with spiky (very high but narrow) barriers in the PEL (which must include infinite-range terms in the Hamiltonian, since in finite-range systems barrier heights can grow at best linearly with barrier width), quantum annealing does much better than CA (Farhi et al., 2002a), as argued by Ray et al. (1989). Quantum adiabatic evolution has been employed earlier to study the SK spin glass in a transverse field; stationary characteristics over a range of the transverse field are calculated by varying the transverse field adiabatically (Lancaster and Ritort, 1997).

It has been argued so far that the advantage of quantum annealing derives mainly from the fact that quantum tunneling can penetrate through very high but narrow barriers, which are hard to jump over thermally. In this way the cost-configuration landscapes are more accessible to local moves. This is a key feature that works even in the case of quantum Monte Carlo methods such as PIMC QA, where one samples only a small section of the configuration space. However, another advantage that quantum mechanics provides is the ability to "sense" the whole configuration space simultaneously through a delocalized wave function. This sensing is greatly handicapped by the presence of random disorder in the system, because the wave function tends to localize in many places, often unable to pick up the deepest well distinctly. This feature may be utilized in searching a golf-course-like PEL, namely, a flat landscape with very deep and narrow wells occurring very rarely. If there is only one deep well, the problem is the spatial version of Grover's problem. As we have seen, if the depth $\chi$ of the well is $O(1)$ (independent of $N$ ), then no more than $O(\sqrt{N})$ speedup can be achieved. This can be interpreted as the inability of an exceedingly large system to sense a given small imperfection. One might want to make the depth of the well large enough that it cannot be scaled away as the system size is increased.

We first consider the case where the well depth varies as $\chi \sim \alpha N$, where $\alpha$ is constant. For a well depth of this order for a spatial search at infinite dimension (the kinetic term is infinite ranged) one gets a stunning result: The evolution time that guarantees any given probability of success becomes independent of $N$ (Das, 2007; see Fig. 4). However, if $\chi$ scales as $\chi \sim N^{\gamma}$, with $\gamma>1$, then the speedup is lost again-a consequence of quantummechanical nonadiabaticity. We formulate the problem in the following way. As in the case of a spatial search discussed above, we denote the state localized at the $i$ th site by $|i\rangle$. All sites except $|w\rangle$ have zero on-site potential energy, while $|w\rangle$ has an on-site potential well of depth $\chi(t)$. The system is embedded in infinite dimensions and thus there is a uniform tunneling term $\Gamma$ between any 

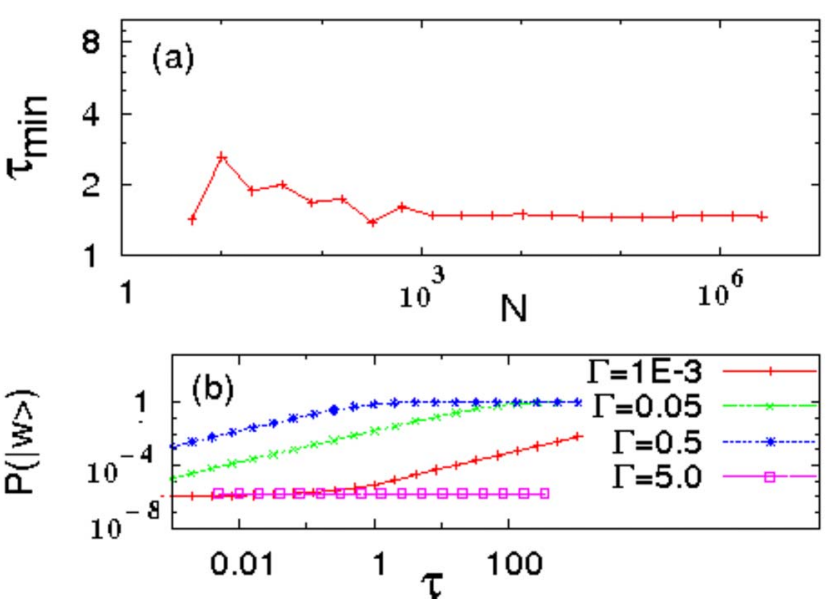

FIG. 4. (Color online) (a) Numerical verification of the $N$ independence on the minimum time $\tau_{\min }$ to achieve success probability $P(|w\rangle)=0.33$. Initially, the system is delocalized equally over all sites and evolves with time according to the time-dependent Schrödinger equation with the Hamiltonian (25). As expected from the exact analytical result for the adiabaticity condition, it is seen that $\tau_{\min }$ becomes independent of $N$. (b) Variation of final probability $P(|w\rangle)$ of finding the state $|w\rangle$ with annealing time $\tau$ for different final values of $\Gamma$, for $N=10^{6}$.

two sites. To perform quantum annealing, we evolve $\chi(t)$ from zero to its final value $\chi_{0}$, keeping $\Gamma$ fixed at some moderate constant value. The total Hamiltonian is given by

$$
\mathcal{H}_{\text {tot }}=-\chi_{0}(1-t / \tau)|w\rangle\left\langle w\left|-\Gamma \sum_{i, j ; i \neq j}\right| i\right\rangle\langle j| .
$$

The time-dependent eigenproblem can be solved for the above Hamiltonian. It can be shown exactly that if $\chi_{0} \sim \alpha N$ then, in the $N \rightarrow \infty$ limit, the adiabatic factor

$$
\frac{\left|\left\langle\dot{\mathcal{H}}_{\text {tot }}\right\rangle\right|_{\max }}{\Delta_{\min }^{2}(t)} \sim \frac{\alpha}{4 \Gamma^{2}},
$$

which means that the run time becomes independent of $N$, and the ground state has $O(1)$ overlap with the target state $|w\rangle$. We also confirm it numerically by evaluating $\tau_{\min }$, which is the minimal $\tau$ required for obtaining a success probability $P(|w\rangle)=0.33$ for different $N$ through many decades. Here we have chosen a moderate constant $\Gamma$, and have evolved the well depth $\chi$ with time. The evolution is computed by solving the timedependent Schrödinger equation numerically, and $\tau_{\min }$ is obtained with an accuracy of $10^{-4}$ by employing a bisection scheme. The results [Fig. 4(a)] show that $P(|w\rangle)$ tends to become independent of $N$ as $N$ becomes larger and larger. This is completely in accordance with the analytical result [see Eq. (26)].

The relaxation behavior for large $N$ for a given annealing time $\tau$ depends on the value of $\Gamma$ [see Fig. 4(b)]. If $\Gamma$ is too small, the system takes a longer time to feel the changes in the landscape, and hence the adiabatic relaxation requires a longer time [the adiabatic factor becomes larger; see Eq. (26)]. On the other hand, if $\Gamma$ is too large, the ground state itself is quite delocalized, and hence the final state, though closer to the ground state, has again a small overlap with the target state $|w\rangle$. The result is best for $\Gamma=0.5$. For higher and lower values of $\Gamma$, the results are worse, as shown in Fig. 4(b). The relaxation behavior is linear with the annealing time $\tau$.

\section{Annealing of a kinetically constrained system}

The adiabatic theorem of quantum annealing assures convergence of a quantum algorithm when one starts with the initial (trivial) ground state of the Hamiltonian and evolves slowly enough so that the system is always in the ground state of the instantaneous Hamiltonian. However, the benefit of tunneling may be extended even in cases where one does not precisely know the eigenstate of the initial Hamiltonian (say, for a given unknown PEL) and hence is unable to start with it. One might rather start with a wave packet (a superposition of many eigenstates of the Hamiltonian) that explores the potential-energy landscape. Quantum tunneling will still allow it to move more easily through the PEL than a classical particle if the landscape has many high but narrow barriers. A semiclassical treatment of such a nonstationary annealing has been discussed in the context of a kinetically constrained system (KCS) (Das et al., 2005).

Here we demonstrate the effectiveness of quantum annealing in the context of certain generalized kinetically constrained systems (KCS) (Fredrickson and Andersen, 1984). KCSs are simple model systems having trivial ground-state structures and static properties, but complex relaxation behavior due to explicit constraints introduced in the dynamics of the system. These systems are important in understanding how much of the slow and complex relaxation behavior of a glass can be attributed to its constrained dynamics alone, ignoring any complexity of its energy landscape structure. In KCSs one can view the constraints as being represented by infinitely-high-energy barriers appearing dynamically.

We now discuss quantum annealing in the context of a $\mathrm{KCS}$, which can be represented by a generalized version of the East model (Jackle and Eisinger, 1991), a onedimensional KCS. We also compare the results with those of thermal annealing in the same system. The original East model is a one-dimensional chain of noninteracting classical Ising ("up-down") spins in a longitudinal field $h$, say, in a downward direction. The ground state of such a system is, trivially, all spins down. A kinetic constraint is introduced in the model by applying the restriction that the $i$ th spin cannot flip if the $(i-1)$ th spin is down. Such a kinetic constraint essentially changes the topology of the configuration space, since the shortest path between any two configurations differing by one or more forbidden flips is increased due to the blockage of the "straight" path consisting of direct flips with dissimilar spins. Further, the constraint becomes more limiting as more spins turn down, as occurs in the late approach to equilibrium. As a result, the re- 
laxation processes have to follow more complex and lengthier paths, giving rise to an exponentially large time scale $\left(\sim e^{1 / T^{2}} ;\right.$ Jackle and Eisinger, 1991).

In the Das model (Das et al., 2005) there is a chain of asymmetric double wells (each with infinite boundary walls), each having a particle localized within it. The asymmetry is due to the energy difference of $2 h$ between the two wells of a double well. The particle in one of the two (asymmetric) wells can change its location to the other well stochastically, due to either the thermal or quantum fluctuations present in the system. The generalized kinetic constraint is introduced by assuming that, if the particle in the $(i-1)$ th double well resides in the lower of the two wells, then there appears a barrier of height $\chi$ and width $a$ between the two wells of the $i$ th double well. In this situation the particle in the $i$ th double well has to cross the barrier in order to change its location from one well to the other. On the other hand, if the particle of the $(i-1)$ th double well is in its upper well, there is no such barrier to cross for the particle to go from one well to the other. Following the approximate mapping done in the case of a symmetric double well (Chakrabarti et al., 1996), this model can be approximately represented by a generalized version of the East model, where each Ising spin is in a local longitudinal field $h$ in the downward direction. The spin at the $i$ th site sees a barrier of height $\chi$ and width $a$ between its two energy states when the $(i-1)$ th spin is down, while no such barrier occurs for the $i$ th spin when the $(i-1)$ th spin is up. This kinetic constraint is the same in both cases irrespective of whether the dynamics is classical or quantum.

When the dynamics of the particle is due to quantum fluctuations, the tunneling probabilities come from the following semiclassical picture of a particle scattering in a double well with infinitely remote outer boundaries. If a particle is put in one of the wells of such a double well with some kinetic energy (actually the expectation value) $\Gamma$, then it will eventually be scattered by the separator (a barrier or step) between the two wells. In such a scattering, there is a finite probability $P$ that the particle manages to go to the other well. We calculate $P$ from the simple picture of a particle scattering by onedimensional potentials as described below. In the thermal case we take simple Boltzmann probabilities for crossing the same barriers. The minimum of the energy of the Ising chain (equivalent to the potential energy of the chain for the double wells) trivially corresponds to the state with all spins down, i.e., aligned along the longitudinal field $h$ (where all particles are in their respective lower wells). To reach the ground state in the quantum case, we start with a large initial value of $\Gamma$ and then reduce it following an exponential schedule given by $\Gamma$ $=\Gamma_{0} \exp \left(-t / \tau_{Q}\right)$. Here $t$ denotes the time and $\tau_{Q}$ sets the effective time scale of annealing. At zero temperature the slow spin-flip dynamics occurs only due to the tunneling (kinetic energy) term $\Gamma$ and hence the system ceases to have any relaxation dynamics in the limit $\Gamma$ $\rightarrow 0$. The barriers are characterized by a height $\chi$ and a

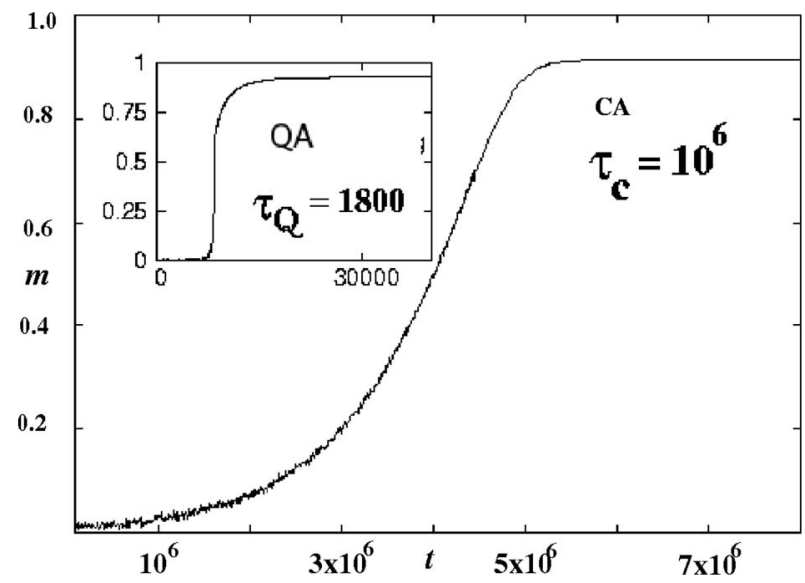

FIG. 5. Comparison between classical and quantum annealing for a chain of $5 \times 10^{4}$ spins (for the same initial disordered configuration with $\left.m_{i} \sim 10^{-3}\right)$. We show results for $\tau_{Q}=1.8$ $\times 10^{2}$ (quantum) and $\tau_{C}=10^{6}$ (classical) with $h=1$; a lower $\tau_{C}$ would not produce substantial annealing. Starting from the same initial values $\Gamma_{0}=T_{0}=100$ (and $g=100$ in the quantum case), we observe that classical annealing requires about $10^{7}$ steps, whereas quantum annealing takes about $10^{4}$ steps to achieve the same final order $m_{f} \sim 0.92$.

width $a$, with the barrier area $g=\chi a$. Similarly, in the thermal case, we start with a high initial temperature $T_{0}$ and reduce it, eventually following an exponentially decreasing temperature schedule given by $T$ $=T_{0} \exp \left(-t / \tau_{C}\right)$, with $\tau_{C}$ the time constant for the thermal annealing schedule. Here, when the $(i-1)$ th spin is down, the flipping probability for the $i$ th spin is $\sim \exp (-\chi / T)$. Otherwise, it flips with probability $P=1$ if it was in the up state and with Boltzmann probability $P=\exp (-h / T)$ if it was in the down state. Here, in the quantum case, the probability of crossing the barrier depends on $g$ so that the barrier width $a$ plays a role, while in the thermal case only $\chi$ sets the crossing time scale, irrespective of $a$.

In the simulation (Das et al., 2005), $N$ Ising spins $\left(S_{i}\right.$ $= \pm 1, i=1, \ldots, N)$ were taken on a linear chain with periodic boundary conditions. The initial spin configuration is taken to be random, so that the magnetization $m=(1 / N) \Sigma_{i} S_{i}$ is practically negligible $\left(m_{i} \approx 0\right)$. We then start with a tunneling field $\Gamma_{0}$ and follow the zerotemperature (semiclassical) Monte Carlo scheme as mentioned above, using the spin-flip probabilities $P$ appropriate for four cases I-IV. Each complete run over the entire lattice is taken as one time unit and, as time progresses, $\Gamma$ is decreased from its initial value $\Gamma_{0}$ according to $\Gamma=\Gamma_{0} e^{-t / \tau} Q$. The results of thermal and quantum annealing are compared in Fig. 5 for the same order of initial value and time constant for $\Gamma$ and $T$ (the barrier height $\chi$ is 1000 in both cases, and $g$ is 100 in the quantum annealing case, or equivalently the barrier width $a$ is on the order of 0.1). It is observed that to achieve a similar degree of annealing (attaining a certain final magnetization $m_{f}$ ), starting from the same disordered configuration, one requires much smaller $\tau_{Q}$ com- 


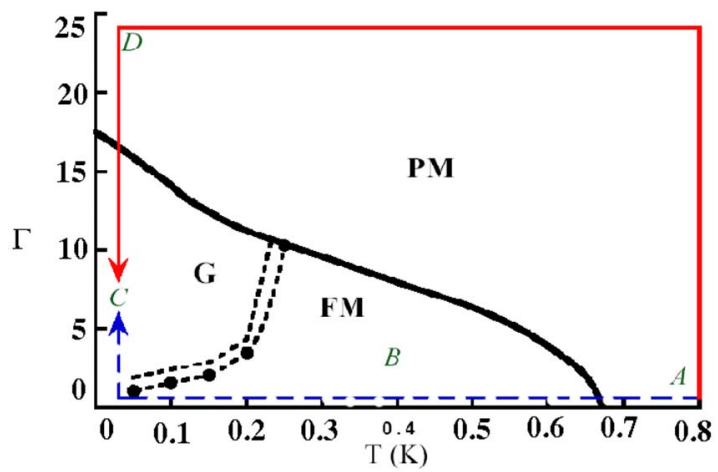

FIG. 6. (Color online) Experimental realization of QA and $\mathrm{CA}$ in $\mathrm{LiHo}_{0.44} \mathrm{Y}_{0.56} \mathrm{~F}_{4}$ illustrated on its phase diagram on the plane of temperature $T$ and transverse field $\Gamma$ (measured by the magnitude of the external laboratory field in kOe). The material behaves as a conventional ferromagnet in the region labeled FM, and shows slow relaxation in the glassy domainwall state labeled $\mathrm{G}$. The two paths of relaxations from an initial point $A$ to a final point $C$ on the phase diagram are shown by arrow-headed lines. Along the classical path $A \rightarrow B$ $\rightarrow C$ (dashed arrow) the transverse field is not applied until the end, so that relaxations observed are purely thermal, whereas, along the quantum path $A \rightarrow D \rightarrow C$ (continuous arrow), there is a segment where the temperature is small enough and the transverse field is high, so that fluctuations are mainly quantum mechanical. Relaxations observed along the quantum path are often found to be much faster than those observed along the classical path at low enough temperature. From Aeppli and Rosenbaum, 2005.

pared to $\tau_{C}$; typically, $\tau_{C} \sim 10^{3} \times \tau_{Q}$ for equivalent annealing (for similar optimal values of final order $m_{f}$ $\sim 0.92$ ). For annealing with final order $m_{f} \sim 1$, we find $\tau_{C} \sim 10^{4} \tau_{Q}$. This comparison depends on the barrier characteristics (the value of $g$ ).

\section{Experimental realization of quantum annealing}

Brooke et al. (1999) showed experimentally (see also Aeppli and Rosenbaum, 2005) that the relaxation behavior in reaching deep inside the glass phase in Fig. 6 depends on the path chosen. A TISG, realized by a sample of $\mathrm{LiHo}_{0.44} \mathrm{Y}_{0.56} \mathrm{~F}_{4}$ in a laboratory transverse field $(\Gamma)$, was taken from a high-temperature paramagnetic to a low-temperature glassy phase following two separate paths in the $\Gamma-T$ plane (see Fig. 6). Along the classical path of cooling (CA), the transverse field was zero throughout, and was switched on only after reaching the final temperature. Quantum cooling (QA), on the other hand, was done in the presence of a high transverse field, which was lowered only on reaching the final temperature. As the sample is cooled, spectroscopy of the sample at different temperatures (during both CA and QA) was done to reveal the distribution of the spin relaxation time scales. The QA produced states whose relaxation was up to 30 times faster than those produced by the CA at low temperature (see Aeppli and Rosenbaum, 2005). This indicates that quantum tunneling is much more effective in exploring the configuration space in the glassy phase than thermal jumps (as indicated in Fig. 1). An experimental realization of quantum adiabatic annealing for three-bit instances of the MAXCUT problem using the NMR technique has also been reported (see Steffen et al., 2003). Here the smoothly varying time-dependent Hamiltonian was realized by quantum simulation (see Nielsen and Chuang, 2000), where a smooth time evolution was achieved approximately (Trotter approximation) through applying a series of discrete unitary operations. The results indicate the existence of an optimal run time of the algorithm.

\section{CONVERGENCE OF QUANTUM ANNEALING ALGORITHMS}

Here we briefly summarize some results derived by Morita and Nishimori $(2006,2007)$ on the convergence of QA algorithms for TIM systems. The results are valid for both the quantum Monte Carlo and the real-time Schrödinger evolution versions of QA.

The Hamiltonian here is the same as given in Eq. (9) with a time dependence in the transverse field $\Gamma=\Gamma(t)$. No assumption regarding either the nature of the distribution of $J_{i j}$ or the spatial dimensionality is required. In order to perform QA at temperature $T$ using the PIMC algorithm, one constructs the Suzuki-Trotter equivalent (see Appendix A.1) $(d+1)$-dimensional classical system of the $d$-dimensional quantum system, and the resulting (classical) system is simulated using a suitable inhomogeneous Markov chain. The transverse field $\Gamma(t)$ is tuned from a high value to zero in the course of the simulation. It can be shown that at the end of the simulation the final distribution converges to the ground state of the classical part of the Hamiltonian irrespective of the initial distribution (strong ergodicity) if

$$
\Gamma(t) \geq M T \tanh ^{-1}\left(\frac{1}{(t+2)^{2 / R L}}\right),
$$

where $M$ is the number of Trotter replicas in the $(d+1)$-dimensional Suzuki-Trotter equivalent system. Here $R$ and $L$ are constants depending on the system size $N$, the spin-flip dynamics appointed for the simulation, the temperature $T$, etc. For large $t$ the bound reduces to

$$
\Gamma(t) \geq M T \frac{1}{(t+2)^{2 / R L}} .
$$

It is remarkable that, in contrast to the inverse logarithmic decay of temperature required for convergence of CA [see Eq. (1)], QA requires only a power-law decay of the transverse field. In this sense, quantum annealing is much faster than the classical annealing for TIM. A similar result can be derived for the more general Hamiltonian (see Morita and Nishimori, 2006). However, the advantage gained here does not change the complexity class of an NP-complete problem, since $R L$ is on the order of $N$ and hence the convergence time is thus still exponential in $N$. 


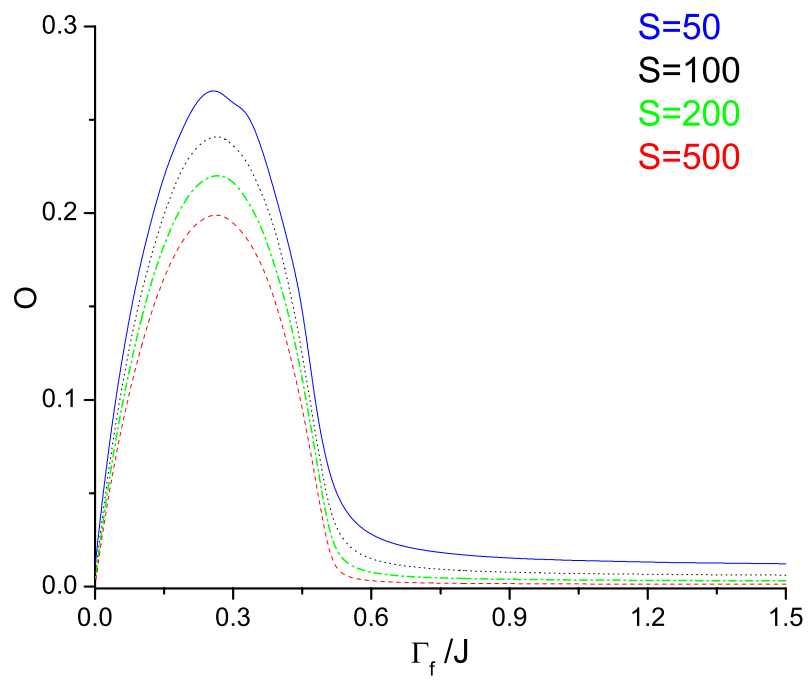

FIG. 7. (Color online) Long-time average (see Appendix A) $O=\left\langle\left(S_{\text {tot }}^{z}\right)^{2}\right\rangle$ as a function of $\Gamma_{f} / J$ for different $S$. The solid, dotted, dash-dotted, and dashed lines represent, respectively, the results for $S=50,100,200$, and 500. $O$ peaks around $\Gamma_{f} / J$ $=0.25$ and the peak value decreases with increasing $S$. For all plots we have chosen $\Gamma_{i} / J=2$. From Das et al., 2006.

For real-time Schrödinger dynamics at $T=0$, the bound for the decay of the transverse field is again of the form

$$
\Gamma(t) \geq(\xi t)^{-1 /(2 N-1)},
$$

where $\xi$ is exponentially small in $N$ for large $N$. Although the dynamics of PIMC QA and real-time Schrödinger evolution are completely different, the power-law bound on the annealing schedule is strikingly similar.

\section{QUANTUM QUENCHING}

Instead of annealing, one can quench a system by reducing the transverse field from $\Gamma_{i}\left(>\Gamma_{c}\right)$ to $\Gamma_{f}\left(<\Gamma_{c}\right)$ and follow the relaxation dynamics after that. Here $\Gamma_{c}$ denotes the quantum critical point for the system. This can help to prepare a state of the system with substantial order in a short time. Recently, quantum quenching dynamics in different systems, in particular for sudden quenching across a quantum critical point, have been studied extensively (Sengupta et al., 2004; Calabrese and Cardy, 2006; Das et al., 2006; Mukherjee et al., 2007; Sengupta et al., 2008). The (exact) results for the afterquench dynamics, following a sudden quench of an infinite-range Ising model in transverse field (Das et al., 2006, see Appendix B), are shown in Fig. 7. The results indicate that for a purely quantum system (small $S$ ), one might reach a nonstationary oscillatory state with a substantial value for the long-time average (over the largest time scale) of order $\left\langle\left(\Sigma S_{i}^{z}\right)^{2}(t)\right\rangle$ if the quenching is done from $\Gamma_{i}>\Gamma_{c}$ to $\Gamma_{f}=\Gamma_{c} / 2$. Thus one gets a state (though nonstationary) which has considerable order, in just onestep quenching. However, if the quenching is carried out with a high but finite rate, one ends up reaching an excited state with topological defects (see Kibble, 1976; Zurek, 1985; Sengupta et al., 2008).

\section{SUMMARY AND DISCUSSIONS}

Unlike gate-based quantum computers (see, e.g., Ekert and Jozsa, 1996; Nielsen and Chuang, 2000; Galindo and Martin-Delgado, 2002), annealing of a physical system toward the optimal state (encoded in the ground state of the final Hamiltonian) in the classical limit naturally achieves analog quantum computation. As discussed here, utilization of quantum-mechanical tunneling through classically localized states in annealing of glasses has opened up this new paradigm for analog quantum computation of hard optimization problems through adiabatic reduction of quantum fluctuations.

We reviewed the recent success in annealing, or optimizing, the cost functions of complex systems, utilizing quantum fluctuations rather than thermal fluctuations (see Santoro and Tosatti, 2006, for a more technical review). As mentioned, following the early indication by Ray et al. (1989) and the pioneering demonstrations, theoretically by Amara et al. (1993), Finnila et al. (1994), Kadowaki and Nishimori (1998), Farhi, Goldstone, Gutmann, et al. (2001), and Santoro et al. (2002), and experimentally by Brooke et al. (1999), the quantum annealing technique has now emerged as a successful technique for optimization of complex cost functions. The literature exploring its success and also its limitations is also considerably developed at present.

These concepts are introduced through discussions on the mapping of such hard problems to classical spinglass problems, discussions on quantum spin glasses, and consequent annealing. The physics of classical spin glasses (see Sec. II) offers us knowledge of the energy or the thermodynamic potential and their landscape structures. Mapping of computationally hard problems like the TSP, etc., to their corresponding classical spin-glass models also helped in understanding their complexity (Sec. II.C). The time scale for tunneling through an energy barrier quantum mechanically involves not only the height of the barrier, but also its width; the narrower the barrier, the faster the tunneling (for constant height). Thermal fluctuations, on the other hand, see only the barrier height. This reduction of tunneling time with barrier width helps in relaxing a quantum system much faster in some cases. This leads to quantum annealing, a framework for constructing general heuristics to find approximate solutions of hard optimization problems. While simulated annealing employs the strategy of slow cooling, physical or in simulations, to find the ground state of glassy systems, quantum annealing employs quantum fluctuations (see Sec. III). As mentioned, this effectively reduces Planck's constant to zero in order to reach the classically optimized (minimum cost) state. This reduction, when done completely adiabatically, guarantees that the system will be found in the ground state of the classical glass at the end (provided there is no crossing of energy levels with the ground state in the 
course of evolution, and one has started initially with the ground state of the Hamiltonian). This has already been realized experimentally (see Sec. III.D), where faster relaxation toward the ground state is achieved by reducing the external field (inducing changes in the tunneling field), rather than by reducing the temperature. In this way analog quantum computation is realized through a novel route. Recently, an equivalence between the adiabatic QA and standard gate-based quantum computation has also been established (Aharonov et al., 2007; Mizel et al., 2007).

\section{ACKNOWLEDGMENTS}

We are grateful to A. Chakrabarti, P. Ray, D. Sen, K. Sengupta, and R. B. Stinchcombe for their collaborations at different stages of this work. We acknowledge G. Aeppli, A. Dutta, H. Nishimori, G. Santoro, P. Sen, and $\mathrm{E}$. Tosatti for useful discussions.

\section{APPENDIX A}

\section{Suzuki-Trotter formalism}

Here we illustrate this formalism by applying it to a TIM having a Hamiltonian

$$
\mathcal{H}=-\Gamma \sum_{i=1}^{N} S_{i}^{x}-\sum_{(i, j)} J_{i j} S_{i}^{z} S_{j}^{z} \equiv \mathcal{H}_{\text {kin }}+\mathcal{H}_{C} .
$$

The canonical partition function of $\mathcal{H}$ reads

$$
Z=\operatorname{Tr} e^{-\left(\mathcal{H}_{\mathrm{kin}}+\mathcal{H}_{C}\right) / T} .
$$

Now we apply the Trotter formula

$$
\exp \left(A_{1}+A_{2}\right)=\lim _{M \rightarrow \infty}\left[\exp \left(A_{1} / M\right) \exp \left(A_{2} / M\right)\right]^{M},
$$

even when $\left[A_{1}, A_{2}\right] \neq 0$. Applying this formula, $Z$ reads

$$
\begin{aligned}
Z= & \lim _{M \rightarrow \infty} \sum_{i}\left\langle s_{i}\right|\left[\exp \left(-\mathcal{H}_{\mathrm{kin}} / M T\right)\right. \\
& \left.\times \exp \left(-\mathcal{H}_{C} / M T\right)\right]^{M}\left|s_{i}\right\rangle .
\end{aligned}
$$

Here $s_{i}$ represents the $i$ th spin configuration of the whole system, and the above summation runs over all such possible configurations denoted by $i$. Now we introduce $M$ identity operators between the product of $M$ exponentials in $Z$, and obtain

$$
\begin{aligned}
Z= & \lim _{M \rightarrow \infty} \operatorname{Tr} \prod_{k=1}^{M}\left\langle S_{1, k} \cdots S_{N, k}\right| \exp \left(\frac{-\mathcal{H}_{\text {kin }}}{M T}\right) \\
& \times \exp \left(\frac{-\mathcal{H}_{C}}{M T}\right)\left|S_{1, k+1} \cdots S_{N, k+1}\right\rangle,
\end{aligned}
$$

and the periodic boundary condition imply $S_{N+1, p}=S_{1, p}$. Now,

$$
\begin{aligned}
& \prod_{k=1}^{M}\left\langle S_{1, k} \cdots S_{N, k}\left|\exp \left(\frac{1}{M T} \sum_{i, j} J_{i j} S_{i}^{z} S_{j}^{z}\right)\right| S_{1, k+1} \cdots S_{N, k+1}\right\rangle \\
& \quad=\exp \left(\sum_{i, j=1}^{N} \sum_{k=1}^{M} \frac{J_{i j}}{M T} S_{i, k} S_{j, k}\right),
\end{aligned}
$$

where $S_{i, k}= \pm 1$ are the eigenvalues of the $S^{z}$ operator (see Hatano and Suzuki, 2005), and

$$
\begin{aligned}
\prod_{k=1}^{M}\left\langle S_{1, k} \cdots S_{N, k}\left|\exp \left(\frac{\Gamma}{M T} \sum_{i} S_{i}^{x}\right)\right| S_{1, k+1} \cdots S_{N, k+1}\right\rangle \\
=\left[\frac{1}{2} \sinh \left(\frac{2 \Gamma}{M T}\right)\right]^{N M / 2} \\
\quad \times \exp \left[\frac{1}{2} \ln \operatorname{coth}\left(\frac{\Gamma}{M T}\right) \sum_{i=1}^{N} \sum_{k=1}^{M} S_{i, k} S_{i, k+1}\right],
\end{aligned}
$$

giving the effective classical Hamiltonian (14), equivalent to the quantum one in Eq. (A1). In the above equation $M$ should be at on the order of $1 / T(\hbar=1)$ for a meaningful comparison of the interaction in the Trotter direction with that in the original Hamiltonian. For $T$ $\rightarrow 0, M \rightarrow \infty$, and the Hamiltonian represents a system of spins in a $(d+1)$-dimensional lattice, because of one extra label $k$ appearing for each spin variable. Thus, corresponding to each single quantum spin variable $S_{i}$ in the original Hamiltonian, we have an array of $M$ classical replica spins $S_{i k}$. The new (timelike) dimension along which these classical spins are spaced is known as the Trotter dimension.

\section{Quantum quenching of a long-range TIM}

Consider a system of spin- $\frac{1}{2}$ objects governed by the Hamiltonian $\mathcal{H}=-(J / N) \Sigma_{i>j}^{N} S_{i}^{z} S_{j}^{z}-\Gamma \Sigma_{i}^{N} S_{i}^{x}$. The Hamiltonian can be rewritten as

$$
\mathcal{H}=-\frac{J}{N}\left(S_{\text {tot }}^{z}\right)^{2}-\Gamma S_{\text {tot }}^{x},
$$

where $S_{\mathrm{tot}}^{z}=\Sigma_{i} S_{i}^{z}, \quad S_{\mathrm{tot}}^{x}=\sum_{i} S_{i}^{x}, \quad$ and a constant $(J / 2 N) \sum_{i}\left(S_{i}^{z}\right)^{2}=J / 8$ from $\mathcal{H}$ in Eq. (A5). The above Hamiltonian can again be cast into the simplified form $\mathcal{H}=\vec{h} \cdot \overrightarrow{S_{\text {tot }}}$, where $\vec{h}=J m \hat{z}-\Gamma \hat{x}$, giving the mean-field (exact in this long-range limit) equation

$$
m \equiv\left\langle S_{\text {tot }}^{z}\right\rangle=\frac{|\vec{h} \cdot \hat{z}|}{|\vec{h}|} \tanh \left(\frac{|\vec{h}|}{T}\right)=\frac{J m}{2 \sqrt{\Gamma^{2}+J^{2} m^{2}}}
$$

at $T=0$, and $\hat{z}$ and $\hat{x}$ denote unit vectors along the $z$ and $x$ directions, respectively. This gives $m=0$ for $\Gamma>\Gamma_{c}$ and $m \neq 0$ for $\Gamma<\Gamma_{c}=J / 2$. Since the model is an infiniteranged one, the mean-field approximation becomes exact, and one can express $\vec{S}$ in terms of its polar components as $\vec{S}=S(\sin \theta \cos \phi, \sin \theta \sin \phi, \cos \theta)$, with $S$ the total angular momentum. One can then utilize the classical equation of motion $\frac{d \vec{S}}{d T}=\vec{S} \times \vec{h}$. Considering the 
above equation for the $z$ and $x$ components, we get

$$
\frac{d \theta}{d t}=\Gamma \sin \theta \quad \text { and } \quad \frac{d \phi}{d t}=-\frac{J}{2} \cos \theta+\Gamma \cot \theta \cos \phi .
$$

Here we have $S=N / 2$. If the system is now quenched from above its quantum critical point $\Gamma>\Gamma_{c}$ finally to $\Gamma_{f}<\Gamma_{c}$, then one can write (see Das et al., 2006), equating the energies of the states with and without any order, respectively, $\Gamma_{f}=(J / 4) \cos ^{2} \theta+\Gamma_{f} \sin \theta \cos \phi$. Using this, one gets from Eq. (A6)

$$
\frac{d \theta}{d t}=\frac{\sqrt{\Gamma_{f}^{2} \sin ^{2} \theta-\left[\Gamma_{f}-(J / 4) \cos ^{2} \theta\right]^{2}}}{\sin \theta} \equiv f(\theta) .
$$

This has zeros (turning points) at $\theta_{1}=\sin ^{-1}\left(\left|1-4 \Gamma_{f} / J\right|\right.$ ) and $\theta_{2}=\pi / 2$. One can therefore obtain $\left\langle\left(S_{\text {tot }}^{z}\right)^{2}\right\rangle=\left\langle\cos ^{2} \theta\right\rangle=\mathcal{N} / \mathcal{D}, \quad$ where $\quad \mathcal{N}=\int_{\theta_{1}}^{\theta_{2}} d \theta \cos ^{2} \theta / f(\theta)$ $=4 \sqrt{8 \Gamma_{f}\left(J-2 \Gamma_{f}\right) / J}$ and $\mathcal{D}=\int_{\theta_{1}}^{\theta_{2}} d \theta / f(\theta)$, giving the behavior shown in Fig. 7.

\section{REFERENCES}

Aeppli, G., and T. F. Rosenbaum, 2005, in Quantum Annealing and Related Optimization Methods, edited by A. Das and B. K. Chakrabarti, Lecture Notes in Physics No. 679 (SpringerVerlag, Heidelberg), pp. 159-169.

Aharonov, D., W. van Dam, J. Kempe, Z. Landau, S. Lloyd, and O. Regev, 2007, SIAM J. Comput. 37166.

Alvarez, J. A., and F. Ritort, 1996, J. Phys. A 29, 7355.

Amara, P., D. Hsu, and J. E. Straub, 1993, J. Phys. Chem. 97, 6715.

Apolloni, B., C. Carvalho, and D. de Falco, 1989, Stochastic Proc. Appl. 33, 233.

Apolloni, B., N. Cesa-Bianchi, and D. de Falco, 1990, in Stochastic Processes, Physics and Geometry, edited by S. Albeverio et al. (World Scientific, Singapore), p. 97.

Barahona, F., 1982, J. Phys. A 15, 3241.

Battaglia, D. A., G. E. Santoro, and E. Tosatti, 2005, Phys. Rev. E 71, 066707.

Battaglia, D. A., L. Stella, O. Zagordi, G. E. Santoro, and E. Tosatti, 2005, in Quantum Annealing and Related Optimization Methods, edited by A. Das and B. K. Chakrabarti, Lecture Notes in Physics No. 679 (Springer-Verlag, Heidelberg), pp. 171-204.

Bearwood, J., J. H. Halton, and J. H. Hammersley, 1959, Proc. Cambridge Philos. Soc. 55, 299.

Bhatt, R. N., 1998, in Spin Glasses and Random Fields, edited by A. P. Young (World Scientific, Singapore), pp. 225-249.

Binder, K., and A. P. Young, 1986, Rev. Mod. Phys. 58, 801.

Bray, A. J., and M. A. Moore, 1984, J. Phys. C 17, L463.

Brooke, J., D. Bitko, T. F. Rosenbaum, and G. Aeppli, 1999, Science 284, 779.

Brooke, J., T. F. Rosenbaum, and G. Aeppli, 2001, Nature (London) 413, 610.

Calabrese, P., and J. Cardy, 2006, Phys. Rev. Lett. 96, 136801. Chakrabarti, B. K., 1981, Phys. Rev. B 24, 4062.

Chakrabarti, B. K., A. Dutta, and P. Sen, 1996, Quantum Ising Phases and Transitions in Transverse Ising Models (SpringerVerlag, Heidelberg).

Chakraborti, A., and B. K. Chakrabarti, 2000, Eur. Phys. J. B
16, 667.

Childs, A. M., and J. Goldstone, 2004, Phys. Rev. A 70, 022314. Das, A., 2007, unpublished (see also Ph.D. thesis, Jadavpur University, Kolkata, 2008).

Das, A., and B. K. Chakrabarti, 2005, Eds., Quantum Annealing and Related Optimization Methods, Lecture Notes in Physics No. 679 (Springer-Verlag, Heidelberg).

Das, A., B. K. Chakrabarti, and R. B. Stinchcombe, 2005, Phys. Rev. E 72, 026701.

Das, A., K. Sengupta, D. Sen, and B. K. Chakrabarti, 2006, Phys. Rev. B 74, 144423.

Dotsenko, V., 2001, Introduction to the Replica Theory of Disordered Statistical Systems (Cambridge University Press, Cambridge, UK).

Edwards, S. F., and P. W. Anderson, 1975, J. Phys. F: Met. Phys. 5, 965.

Ekert, A., and R. Jozsa, 1996, Rev. Mod. Phys. 68, 733.

Farhi, E., J. Goldstone, and S. Gutmann, 2002a, e-print arXiv:quant-ph/0201031.

Farhi, E., J. Goldstone, and S. Gutmann, 2002b, e-print arXiv:quant-ph/0208135.

Farhi, E., J. Goldstone, S. Gutmann, J. Lapan, A. Ludgren, and D. Preda, 2001, Science 292, 472.

Farhi, E., J. Goldstone, S. Gutmann, and M. Sipser, 2000, e-print arXiv:quant-ph/0001106.

Farhi, E., and S. Gutmann, 1998, Phys. Rev. A 57, 2403.

Finnila, A. B., M. A. Gomez, C. Sebenik, C. Stenson, and D. J. Doll, 1994, Chem. Phys. Lett. 219, 343.

Fisher, D. S., and D. A. Huse, 1986, Phys. Rev. Lett. 56, 1601. Fredrickson, G. H., and H. C. Andersen, 1984, Phys. Rev. Lett. 53, 1244.

Fu, Y., and P. W. Anderson, 1986, J. Phys. A 19, 1620.

Galindo, A., and M. A. Martin-Delgado, 2002, Rev. Mod. Phys. 74, 347.

Garey, M. R., and D. S. Johnson, 1979, Computers and Intractability: Guide to the Theory of NP-Completeness (Freeman, San Francisco).

Gaviro, P. S., J. J. Ruiz-Lorenzo, and A. Tarancon, 2006, J. Phys. A 39, 8567.

Geman, S., and D. Geman, 1984, IEEE Trans. Pattern Anal. Mach. Intell. 6, 721.

Goldschmidt, Y. Y., and P.-Y. Lai, 1990, Phys. Rev. Lett. 64, 2467.

Grover, L. K., 1997, Phys. Rev. Lett. 79, 325.

Guo, M., R. N. Bhatt, and D. A. Huse, 1994, Phys. Rev. Lett. 72, 4137.

Hartmann, A., and H. Rieger, 2002, Optimization in Physics (Wiley VCH, Darmstadt).

Hatano, N., and M. Suzuki, 2005, in Quantum Annealing and Related Optimization Methods, edited by A. Das and B. K. Chakrabarti, Lecture Notes in Physics No. 679 (SpringerVerlag, Heidelberg), pp. 37-67.

Huse, D. A., and D. S. Fisher, 1986, Phys. Rev. Lett. 57, 2203. Inoue, J.-I., 2005, in Quantum Annealing and Related Optimization Methods, edited by A. Das and B. K. Chakrabarti, Lecture Notes in Physics No. 679 (Springer-Verlag, Heidelberg), pp. 259-296.

Ishii, H., and T. Yamamoto, 1985, J. Phys. C 18, 6225.

Jackle, J., and S. Eisinger, 1991, Z. Phys. B: Condens. Matter 84, 115.

Kadowaki, T., and H. Nishimori, 1998, Phys. Rev. E 58, 5355. Kibble, T. W. B., 1976, J. Phys. A 9, 1387.

Kim, D.-H., and J.-J. Kim, 2002, Phys. Rev. B 66, 054432. 
Kirkpatrick, S., C. D. Gelatt, and M. P. Vecchi, 1983, Science 220, 671.

Krzakala, F., J. Houdayer, E. Marinari, O. C. Martin, and G. Parisi, 2001, Phys. Rev. Lett. 87, 197204.

Lancaster, D., and F. Ritort, 1997, J. Phys. A 30, L41.

Marinari, E., G. Parisi, J. J. Ruiz-Lorenzo, and F. Zuliani, 1998, Phys. Rev. Lett. 82, 5176.

Martoňák, R., G. E. Santoro, and E. Tosatti, 2002, Phys. Rev. B 66, 094203.

Martoňák, R., G. E. Santoro, and E. Tosatti, 2004, Phys. Rev. E 70, 057701.

Mezard, M., G. Parisi, and M. A. Virasoro, 1987, Spin Glass Theory and Beyond, Lecture Note in Physics No. 9 (World Scientific, Singapore), and references therein.

Miller, J., and D. A. Huse, 1993, Phys. Rev. Lett. 70, 3147.

Mizel, A., D. A. Lidar, and M. Mitchell, 2007, Phys. Rev. Lett. 99, 070502.

Monasson, R., R. Zecchina, S. Kirkpatrick, B. Selman, and L. Troyansky, 1999, Nature (London) 400, 133.

Moore, M. A., H. Bokil, and B. Drossel, 1998, Phys. Rev. Lett. 81, 4252.

Morita, S., 2007, J. Phys. Soc. Jpn. 76, 104001.

Morita, S., and H. Nishimori, 2006, J. Phys. A 39, 13903.

Morita, S., and H. Nishimori, 2007, J. Phys. Soc. Jpn. 76, 064002.

Mukherjee, V., U. Divakaran, A. Dutta, and D. Sen, 2007, Phys. Rev. B 76, 174303.

Nielsen, M. A., and I. L. Chuang, 2000, Quantum Computation and Quantum Information (Cambridge University Press, Cambridge, UK).

Nishimori, H., 2001, Statistical Physics of Spin Glasses and Information Processing: An Introduction (Oxford University Press, Oxford).

Papadimitriou, C. H., and K. Steiglitz, 1998, Combinatorial Optimization: Algorithm and Complexity (Dover, New York). Parisi, G., 1980, J. Phys. A 13, 1101.

Percus, A., and O. C. Martin, 1996, Phys. Rev. Lett. 76, 1188.

Ray, P., B. K. Chakrabarti, and A. Chakrabarti, 1989, Phys. Rev. B 39, 11828.

Rieger, H., 2005, in Quantum Annealing and Related Optimization Methods, edited by A. Das and B. K. Chakrabarti, Lecture Notes in Physics No. 679 (Springer-Verlag, Heidelberg), pp. 69-97.

Roland, J., and N. J. Cerf, 2001, Phys. Rev. A 65, 042308.
Rodriguez-Laguna, J., 2007, J. Stat. Mech.: Theory Exp. 2007, P05008.

Sachdev, S., 1999, Quantum Phase Transitions (Cambridge University Press, Cambridge, UK).

Santoro, G. E., R. Martoňák, E. Tosatti, and R. Car, 2002, Science 295, 2427.

Santoro, G. E., and E. Tosatti, 2006, J. Phys. A 39, R393.

Santoro, G. E., and E. Tosatti, 2007, Nat. Phys. 3, 593.

Sarandy, M. S., L.-A. Wu, and D. A. Lidar, 2004, Quantum Inf. Process. 3, 331.

Sarjala, M., V. Petäjä, and M. Alava, 2006, J. Stat. Mech.: Theory Exp. 2006, PO 1008.

Sengupta, K., S. Powell, and S. Sachdev, 2004, Phys. Rev. A 69, 053616.

Sengupta, K., D. Sen, and S. Mondal, 2008, Phys. Rev. Lett. 100, 077204.

Sherrington, D., and S. Kirkpatrick, 1975, Phys. Rev. Lett. 35, 1792.

Schechter, M., and N. Laflorencie, 2006, Phys. Rev. Lett. 97, 137204.

Silevitch, D. M., D. Bitko, J. Brooke, S. Ghosh, G. Aeppli, and T. F. Rosenbaum, 2007, Nature (London) 448, 567.

Somma, R. D., C. D. Batista, and G. Ortiz, 2007, Phys. Rev. Lett. 99, 030603.

Suzuki, S., H. Nishimori, and M. Suzuki, 2007, Phys. Rev. E 75, 051112.

Suzuki, S., and M. Okada, 2005a, J. Phys. Soc. Jpn. 74, 1649. Suzuki, S., and M. Okada, 2005b, in Quantum Annealing and Related Optimization Methods, edited by A. Das and B. K. Chakrabarti, Lecture Notes in Physics No. 679 (SpringerVerlag, Heidelberg).

Steffen, M., v. W. Dam, T. Hogg, G. Breyta, and I. Chuang, 2003, Phys. Rev. Lett. 90, 067903.

Stella, L., G. Santoro, and E. A. Tosatti, 2005, Phys. Rev. B 72, 014303.

Thirumalai, D., Q. Li, and T. R. Kirkpatrick, 1989, J. Phys. A 22, 2339.

Wu, W., D. Bitko, T. F. Rosenbaum, and G. Aeppli, 1993, Phys. Rev. Lett. 71, 1919.

Wu, W., B. Ellman, T. F. Rosenbaum, G. Aeppli, and D. H. Reich, 1991, Phys. Rev. Lett. 67, 2076.

Ye, J., S. Sachdev, and N. Read, 1993, Phys. Rev. Lett. 70, 4011. Zurek, W. H., 1985, Nature (London) 317, 505. 